\title{
Trajectory-Tracking and Path-Following of Underactuated Autonomous Vehicles with Parametric Modeling Uncertainty
}

\author{
A. Pedro Aguiar, Member, IEEE, and João P. Hespanha, Senior Member, IEEE
}

\begin{abstract}
We address the problem of position trajectorytracking and path-following control design for underactuated autonomous vehicles in the presence of possibly large modeling parametric uncertainty. For a general class of vehicles moving in either two or three-dimensional space, we demonstrate how adaptive switching supervisory control can be combined with a nonlinear Lyapunov-based tracking control law to solve the problem of global boundedness and convergence of the position tracking error to a neighborhood of the origin that can be made arbitrarily small. The desired trajectory does not need to be of a particular type (e.g., trimming trajectories) and can be any sufficiently smooth bounded curve parameterized by time. We also show how these results can be applied to solve the path-following problem, in which the vehicle is required to converge to and follow a path, without a specific temporal specification. We illustrate our design procedures through two vehicle control applications: a hovercraft (moving on a planar surface) and an underwater vehicle (moving in three-dimensional space). Simulations results are presented and discussed.
\end{abstract}

Index Terms-Supervisory adaptive control, path-following, trajectory-tracking, underactuated autonomous vehicles.

\section{INTRODUCTION}

$\mathbf{T}$ HE past few decades have witnessed an increased research effort in the area of motion control of autonomous vehicles. A typical motion control problem is trajectorytracking, which is concerned with the design of control laws that force a vehicle to reach and follow a time parameterized reference (i.e., a geometric path with an associated timing law). The degree of difficulty involved in solving this problem is highly dependent on the configuration of the vehicle. For fully actuated systems, the trajectory-tracking problem is now reasonably well understood.

For underactuated vehicles, i.e., systems with fewer actuators than degrees-of-freedom ${ }^{1}$, trajectory-tracking is still

Research supported in part by the National Science Foundation under Grant No. ECS-0093762, project MAYA-Sub of the AdI (PT), project GREX / CECIST (Contract No. 035223) of the Commission of the European Communities, and by the FCT-ISR/IST plurianual funding through the POS_C Program that includes FEDER funds.

A. Pedro Aguiar was with the Center for Control Engineering and Computation, University of California, Santa Barbara, CA 93106-9560, USA. He is now with the Institute for Systems and Robotics, Instituto Superior Tenico, Lisbon, Portugal (e-mail: pedro@isr.ist.utl.pt).

J. P. Hespanha is with Center for Control Engineering and Computation, University of California, Santa Barbara, CA 93106-9560, USA. (e-mail: hespanha@ece.ucsb.edu)

${ }^{1}$ The following definition of underactuated mechanical systems is adapted from [1], [2]. Consider the affine mechanical system described by

$$
\ddot{q}=f(q, \dot{q})+G(q) u,
$$

an active research topic. The study of these systems is motivated by the fact that it is usually costly and often not practical to fully actuate autonomous vehicles due to weight, reliability, complexity, and efficiency considerations. Typical examples of underactuated systems include wheeled robots, hovercraft, spacecraft, aircraft, helicopters, missiles, surface vessels, and underwater vehicles. The tracking problem for underactuated vehicles is especially challenging because most of these systems are not fully feedback linearizable and exhibit nonholonomic constraints. The reader is refereed to [3] for a survey of these concepts and to [4] for a framework to study the controllability and the design of motion algorithms for underactuated Lagrangian systems on Lie groups.

The classical approach for trajectory-tracking of underactuated vehicles utilizes local linearization and decoupling of the multi-variable model to steer the same number of degrees of freedom as the number of available control inputs, which can be done using standard linear (or nonlinear) control methods. Alternative approaches include the linearization of the vehicle error dynamics around trajectories that lead to a time-invariant linear system (also known as trimming trajectories) combined with gain scheduling and/or Linear Parameter Varying (LPV) design methodologies [5]-[7]. The basic limitation of these approaches is that stability is only guaranteed in a neighborhood of the selected operating points. Moreover, performance can suffer significantly when the vehicle executes maneuvers that emphasize its nonlinearity and cross-couplings. A different approach is to use output feedback linearization methods, [8][10]. The major challenge in this approach is that a straightforward application of this methodology, which in general involves dynamic inversion, is not always possible because certain involutivity conditions must hold [11]. In addition, even when dynamic inversion is possible, the resulting controller may not render the zero-dynamics stable.

Nonlinear Lyapunov-based designs can overcome some of the limitations mentioned above. Several examples of nonlinear trajectory-tracking controllers for marine underactuated vehicles have been reported in the literature [12]-[19]. Typically, tracking problems for autonomous vehicles are solved by designing control laws that make the vehicles track pre-

where $q$ is a vector of independent generalized coordinates, $f$ a vector field that captures the dynamics of the system, $G$ the input matrix, and $u$ the vector of generalized inputs. The system (1) is underactuated if the rank of $G$ is smaller than the dimension of $q$, i.e., the generalized inputs are not able to instantaneously set the accelerations in all directions of the configuration space. 
specified feasible "state-space" trajectories, i.e., trajectories that specify the time evolution of the position, orientation, as well as the linear and angular velocities, all consistent with the vehicles' dynamics, [8], [13], [15]-[20], even through in practical applications one often only needs to track a desired position. This approach suffers from the drawback that usually the vehicles' dynamics exhibit complex nonlinear terms and significant uncertainty, which makes the task of computing a feasible trajectory difficult.

It is relevant to point out that most of the results mentioned above only solve the problem in the horizontal plane. Only a few authors have tackled this control problems in three dimensional space. The reason might be that the vehicle's dynamics become more complex and the number of degree of freedom that are not directly actuated typically increases, making the control design more involved. For example, for an underactuated underwater vehicle, the dynamics include sway and heave velocities that generate nonzero angles of sideslip and attack.

Motivated by the above considerations, we propose a solution to the trajectory-tracking problem for underactuated vehicles in both two and three-dimensional spaces. In this paper we are especially interested in situations for which there is parametric uncertainty in the model of the vehicle. Typical parameters for which this uncertainty is high, include mass and added mass for underwater vehicles which may be subject to large variations according to the payload configuration, and friction coefficients that are usually strongly dependent on the environmental conditions. The main contribution of the paper is the design of an adaptive supervisory control algorithm that combines logic-based switching [21] with iterative Lyapunov-based techniques such as integrator backstepping [22]. The classical approach to adaptive control relies solely on continuous tuning [22]-[24]. This approach has some inherent limitations that can be overcome by hybrid adaptive algorithms based on switching and logic [25]. The basic idea behind supervisory control [21], [26]-[30] is to design a suitable family of candidate controllers. Each controller is designed for an admissible nominal model of the process, and a supervision logic orchestrates the switching among the candidate controllers, deciding, at each instant of time, the candidate feedback controller that is more adequate. In order to guarantee stability and avoid chattering, a form of hysteresis is employed. We prove that the adaptive controller solves the problem of global boundedness and convergence of the position tracking error to a neighborhood of the origin that can be made arbitrarily small in the presence of possible large parametric uncertainty. The adaptive supervisory controller does not require persistence of excitation which sets it apart from most parameter estimation algorithms. In the control design, we take into account that the vehicle may have nonnegligible dynamics and may undergo complex motions and exhibit large angles of attack and sideslip, which prevents us from using simple extensions of common control designs for wheeled robots where the total velocity vector is aligned with the vehicles main axis. Also, the desired trajectory does not need to be a trimming trajectory and can be any sufficiently smooth time-varying bounded curve, including the degenerate case of a constant trajectory (set-point). The class of vehicles for which the design procedure is applicable is quite general and includes any vehicle modeled as a rigidbody subject to a controlled force and either one controlled torque if it is only moving on a planar surface or two or three independent control torques for a vehicle moving in three dimensional space. Furthermore, contrary to most of the approaches described above, the controller proposed does not suffer from geometric singularities due to the parameterization of the vehicle's rotation matrix. This is possible because the attitude control problem is formulated directly in the group of rotations $S O(3)$. The literature on designing tracking control laws for underactuated vehicles directly in the configuration manifold (avoiding in this way geometric singularities) is relatively scarce. Noteworthy examples include [20], [31].

Another contribution of this paper is the application of these results to solve the path-following motion control problem. In path-following, the vehicle is required to converge to and follow a path that is specified without a temporal law [32][36]. Pioneering work in this area for wheeled mobile robots is described in [32]. In [34], Samson addressed the pathfollowing problem for a car pulling several trailers. More recently, Altafini [36] describes a path-following controller for a $n$ trailer vehicle that provides local asymptotic stability for a path of nonconstant curvature. Path-following controllers for aircraft and marine vehicles have been reported in [6], [9], [37]-[39]. Using the approach suggested by Hauser and Hindman [37], an output maneuvering controller was proposed in [39] for a class of strict feedback nonlinear processes and applied to path-following of fully actuated ships. The underlying assumption in path-following is that the vehicle's forward speed tracks a desired speed profile, while the controller acts on the vehicle's orientation to drive it to the path. Typically, in path-following, smoother convergence to the path is achieved and the control signals are less likely pushed into saturation, when compared to trajectory-tracking. In fact, in [40], [41], we highlight a fundamental difference between path-following and standard trajectory-tracking by demonstrating that performance limitations due to unstable zero-dynamics can be removed in the path-following problem. Inspired by these ideas, we solve the path-following problem by decomposing it into two subproblems: i) a geometric task, which consists of converging the vehicle to and remaining inside a tube centered around the desired path, and ii) a dynamic assignment task, which assigns a speed profile to the path.

In Section II we describe the dynamic model for the class of underactuated autonomous vehicles considered in the paper and formulate the trajectory-tracking and path-following control problems. As a preliminary material for the subsequent sections, Section III presents a nonlinear control law to solve the tracking problem and discusses the stability of the resulting closed-loop. At this point it is assumed that there is no parametric uncertainty. Sections IV and V present the main results of the paper. In Section IV, a solution to the trajectorytracking is proposed using an estimator-based supervisory controller, and in Section V an extension is made to solve the path-following problem. In Section VI, we illustrate our 
design methodologies in the context of two vehicle control applications: a hovercraft (moving on a planar surface) and an underwater vehicle (moving in three-dimensional space). The designs are validated through computer simulations. The paper concludes with a summary of the results and suggestions for further research.

A subset of the results reported here were presented in [42][44].

Notation: Throughout this paper, given a matrix $A, A^{\prime}$ denotes its transpose, $\lambda_{\min }(A), \lambda_{\max }(A)$ are the minimum and maximum eigenvalues of $A$, respectively. Given two vectors $v_{1} \in \mathbb{R}^{n_{1}}, v_{2} \in \mathbb{R}^{n_{2}}$, we denote by $\operatorname{col}\left(v_{1}, v_{2}\right)$ the vector $\left(v_{1}^{\prime}, v_{2}^{\prime}\right)^{\prime} \in \mathbb{R}^{n_{1}+n_{2}}$. The Euclidean norm is denoted by $\|\cdot\|$ and the spectral norm by $\|\cdot\|_{2}$. A piecewise continuous function $g:[0, T) \rightarrow \mathbb{R}^{n}, T \in(0, \infty]$ is in $\mathcal{L}_{i}, i$ being a positive integer, if $\int_{0}^{T}\|g(\tau)\|^{i} d \tau<c$ for some constant $c$.

\section{Problem StATEMEnT}

Consider an underactuated vehicle modeled as a rigid body subject to external forces and torques. Let $\{\mathcal{I}\}$ be an inertial coordinate frame and $\{\mathcal{B}\}$ a body-fixed coordinate frame whose origin is located at the center of mass of the vehicle. The configuration $(R, p)$ of the vehicle is an element of the Special Euclidean group $S E(3):=S O(3) \times \mathbb{R}^{3}$, where $R \in S O(3):=\left\{R \in \mathbb{R}^{3 \times 3}: R R^{\prime}=I_{3}, \operatorname{det}(R)=+1\right\}$ is a rotation matrix that describes the orientation of the vehicle by mapping body coordinates into inertial coordinates, and $p \in \mathbb{R}^{3}$ is the position of the origin of $\{\mathcal{B}\}$ in $\{\mathcal{I}\}$. Denoting by $v \in \mathbb{R}^{3}$ and $\omega \in \mathbb{R}^{3}$ the linear and angular velocities of the vehicle relative to $\{\mathcal{I}\}$ expressed in $\{\mathcal{B}\}$, respectively, the following kinematic relations apply:

$$
\begin{aligned}
\dot{p} & =R v \\
\dot{R} & =R S(\omega)
\end{aligned}
$$

where $S(\cdot)$ is a function from $\mathbb{R}^{3}$ to the space of skewsymmetric matrices $\mathcal{S}:=\left\{M \in \mathbb{R}^{3 \times 3}: M=-M^{\prime}\right\}$ defined by

$$
S(x):=\left[\begin{array}{ccc}
0 & -x_{3} & x_{2} \\
x_{3} & 0 & -x_{1} \\
-x_{2} & x_{1} & 0
\end{array}\right], \quad \forall x:=\left(x_{1}, x_{2}, x_{3}\right)^{\prime} \in \mathbb{R}^{3} .
$$

We consider here underactuated vehicles with dynamic equations of motion of the following form:

$$
\begin{aligned}
\mathbf{M} \dot{v} & =-S(\omega) \mathbf{M} v+f_{v}(v, p, R)+g_{v} u_{v} \\
\mathbf{J} \dot{\omega} & =-S(v) \mathbf{M} v-S(\omega) \mathbf{J} \omega+f_{\omega}(v, \omega, p, R)+G_{\omega} u_{\omega}
\end{aligned}
$$

where $\mathbf{M} \in \mathbb{R}^{3 \times 3}$ and $\mathbf{J} \in \mathbb{R}^{3 \times 3}$ denote constant symmetric positive definite mass and inertia matrices; $u_{v} \in \mathbb{R}$ and $u_{\omega} \in \mathbb{R}^{3}$ denote the control inputs, which act upon the system through a constant nonzero vector $g_{v} \in \mathbb{R}^{3}$ and a constant nonsingular matrix ${ }^{2} G_{\omega} \in \mathbb{R}^{3 \times 3}$, respectively; the terms $-S(\omega) \mathbf{M} v$ in (3a) and $-S(v) \mathbf{M} v-S(\omega) \mathbf{J} \omega$ in (3b) are the rigid-body Coriolis terms, and the $\mathcal{C}^{1}$ functions $f_{v}(\cdot), f_{\omega}(\cdot)$ represent all the remaining forces and torques acting on the body. For the special case of an underwater vehicle, $\mathbf{M}$ and $\mathbf{J}$ also include the so-called hydrodynamic added-mass $M_{A}$ and

\footnotetext{
${ }^{2}$ See Remark 4 for the special case of $G_{\omega} \in \mathbb{R}^{3 \times 2}$.
}

added-inertia $J_{A}$ matrices, respectively, i.e., $\mathbf{M}=M_{R B}+M_{A}$, $\mathbf{J}=J_{R B}+J_{A}$, where $M_{R B}$ and $J_{R B}$ are the rigid-body mass and inertia matrices, respectively.

For an underactuated vehicle restricted to moving on a planar surface, the same equations of motion (2)-(3) apply without the first two right-hand-side terms in (3b). Also, in this case, $(R, p) \in S E(2), v \in \mathbb{R}^{2}, \omega \in \mathbb{R}, g_{v} \in \mathbb{R}^{2}, G_{\omega} \in \mathbb{R}$, $u_{\omega} \in \mathbb{R}$, with all the other terms in (3) having appropriate dimensions, and the skew-symmetric matrix $S(\omega)$ is given by $S(\omega)=\left(\begin{array}{cc}0 & -\omega \\ \omega & 0\end{array}\right)$. For simplicity, in what follows, we restrict our attention to the three-dimensional case. However, all results are directly applicable to the two-dimensional case, as will be illustrated in Section VI-A for the control of a Hovercraft.

Remark 1: The vehicle dynamic model (3) does not allow $f_{v}$ to depend on $\omega$. This was done in part to simplify the analysis and also because in many vehicles this dependance is not present as is the case of the Hovercraft and the AUV described in Section VI. The methodology presented here still applies for the more general case if the dependence on $\omega$ is in the form $f_{v}(\cdot)=f_{v_{1}}(v, p, R)+f_{v_{2}}(v, p, R) \omega$, provided that $f_{v_{2}}$ is bounded or that a suitable rank condition holds. For details see Property 1 in the Appendix and [42], [44].

The problems considered in this paper can be stated as follows:

Trajectory-tracking problem: Let $p_{d}(t):[0, \infty) \rightarrow \mathbb{R}^{3}$ be a given sufficiently smooth time-varying desired trajectory with its time-derivatives bounded. Design a controller such that all the closed-loop signals are bounded and the tracking error $\left\|p(t)-p_{d}(t)\right\|$ converges to a neighborhood of the origin that can be made arbitrarily small.

Path-following problem: Let $p_{d}(\gamma) \in \mathbb{R}^{3}$ be a desired path parameterized by $\gamma \in \mathbb{R}$ and $v_{r}(\gamma) \in \mathbb{R}$ a desired speed ${ }^{3}$ assignment. Suppose also that $p_{d}(\gamma)$ is sufficiently smooth with respect to $\gamma$ and its derivatives (with respect to $\gamma$ ) are bounded. Design feedback control laws for $u_{v}, u_{\omega}$, and $\ddot{\gamma}$ such that all the closed-loop signals are bounded, the position of the vehicle converges to and remains inside a tube centered around the desired path that can be made arbitrarily thin, i.e., $\| p(t)-$ $p_{d}(\gamma(t)) \|$ converges to a neighborhood of the origin that can be made arbitrarily small, and the vehicle satisfies a desired speed assignment $v_{r}$ along the path, i.e., the speed error $\dot{\gamma}(t)-$ $v_{r}(\gamma(t))$ can be confined to an arbitrarily small ball.

\section{TRAJECTORY-TRACKING CONTROLLER DESIGN}

\section{A. Controller design}

This section proposes a Lyapunov-based control law to solve the trajectory-tracking problem assuming that there is no parametric uncertainty. For the sake of clarity, controlLyapunov functions are introduced iteratively borrowing from the techniques of backstepping [22].

Step 1. Coordinate transformation: Consider the global diffeomorphic coordinate transformation

$$
e:=R^{\prime}\left(p-p_{d}\right)
$$

\footnotetext{
${ }^{3}$ For simplicity of presentation it will be assumed that the speed assignment $v_{r}(\gamma) \in \mathbb{R}$ does not depend directly on time $t$.
} 
which expresses the tracking error $p-p_{d}$ in the body-fixed frame. The dynamic equation of the body-fixed tracking error $e$ is given by

$$
\dot{e}=-S(\omega) e+v-R^{\prime} \dot{p}_{d} .
$$

Step 2. Convergence of e: We start by defining the controlLyapunov function

$$
V_{1}:=\frac{1}{2} e^{\prime} e
$$

and computing its time derivative to obtain

$$
\dot{V}_{1}=e^{\prime}\left[v-R^{\prime} \dot{p}_{d}\right] .
$$

We can regard $v$ as a virtual control that one would use to make $\dot{V}_{1}$ negative. This could be achieved, by setting $v$ equal to $R^{\prime} \dot{p}_{d}-k_{e} \mathbf{M}^{-1} e$, for some positive constant $k_{e}$. To accomplish this we introduce the error variable

$$
z_{1}:=v-R^{\prime} \dot{p}_{d}+k_{e} \mathbf{M}^{-1} e
$$

that we would like to drive to zero, and re-write (4) as

$$
\dot{V}_{1}=-k_{e} e^{\prime} \mathbf{M}^{-1} e+e^{\prime} z_{1} .
$$

Step 3. Backstepping for $z_{1}$ : After straightforward algebraic manipulations, the dynamic equation of the error $z_{1}$ can be written as

$$
\mathbf{M} \dot{z}_{1}=S\left(\mathbf{M} z_{1}\right) \omega+\Gamma(\cdot) \omega+g_{v} u_{v}+h(\cdot)
$$

where

$$
\Gamma\left(R, \dot{p}_{d}\right):=S\left(\mathbf{M} R^{\prime} \dot{p}_{d}\right)-\mathbf{M} S\left(R^{\prime} \dot{p}_{d}\right)
$$

and $h\left(e, p, R, v, z_{1}, \ddot{p}_{d}\right):=f_{v}(v, p, R)-\mathbf{M} R^{\prime} \ddot{p}_{d}+k_{e} z_{1}-$ $k_{e}^{2} \mathbf{M}^{-1} e$. It turns out that it will not always be possible to drive $z_{1}$ to zero. We need to explore the coupling of the translation dynamics with the rotational inputs. To this effect, we will drive $z_{1}$ to a constant design vector $\delta \in \mathbb{R}^{3}$. To achieve this we define $\varphi:=z_{1}-\delta$ as a new error variable that we will drive to zero and consider the augmented control-Lyapunov function

$$
V_{2}:=V_{1}+\frac{1}{2} \varphi^{\prime} \mathbf{M}^{2} \varphi=\frac{1}{2} e^{\prime} e+\frac{1}{2} \varphi^{\prime} \mathbf{M}^{2} \varphi .
$$

The time derivative of $V_{2}$ can be written as

$$
\dot{V}_{2}=-k_{e} e^{\prime} \mathbf{M}^{-1} e+e^{\prime} \delta+\varphi^{\prime}(\mathbf{M B}(\cdot) \zeta+\mathbf{M} h(\cdot)+e)
$$

where

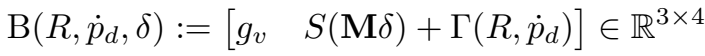

$$
\begin{aligned}
& \zeta:=\operatorname{col}\left(u_{v}, \omega\right) \in \mathbb{R}^{4} .
\end{aligned}
$$

In Appendix (cf. Property 1), we show that the matrix B can always be made full-rank by choosing a suitable $\delta$. One can now regard $\zeta$ as a virtual control (actually its first component is already a "real" control) that one would like to use to make $\dot{V}_{2}$ negative. This could be achieved, by setting $\zeta$ equal to

$$
\alpha:=\mathrm{B}^{\prime}\left(\mathrm{BB}^{\prime}\right)^{-1}\left(-h(\cdot)-\mathbf{M}^{-1} e-\mathbf{M}^{-1} K_{\varphi} \varphi\right),
$$

where $K_{\varphi} \in \mathbb{R}^{3 \times 3}$ is a symmetric positive definite matrix. To accomplish this we set $u_{v}$ to be equal to the first entry of $\alpha$, i.e.,

$$
u_{v}=\left[\begin{array}{ll}
1 & 0_{1 \times 3}
\end{array}\right] \alpha
$$

and introduce the error variable

$$
z_{2}:=\omega-\left[\begin{array}{cc}
0_{3 \times 1} & I_{3 \times 3}
\end{array}\right] \alpha
$$

that one would like to set to zero. We can now re-write (7), with $u_{v}$ given by (9), as

$\dot{V}_{2}=-k_{e} e^{\prime} \mathbf{M}^{-1} e+e^{\prime} \delta-\varphi^{\prime} K_{\varphi} \varphi+\varphi^{\prime} \mathbf{M}[S(\mathbf{M} \delta)+\Gamma(\cdot)] z_{2}$.

Step 4. Backstepping for $z_{2}$ : Consider now a third controlLyapunov function given by

$$
V_{3}:=V_{2}+\frac{1}{2} z_{2}^{\prime} \mathbf{J} z_{2}=\frac{1}{2} e^{\prime} e+\frac{1}{2} \varphi^{\prime} \mathbf{M}^{2} \varphi+\frac{1}{2} z_{2}^{\prime} \mathbf{J} z_{2} .
$$

Computing its time derivative one obtains

$$
\begin{aligned}
\dot{V}_{3}= & -k_{e} e^{\prime} \mathbf{M}^{-1} e+e^{\prime} \delta-\varphi^{\prime} K_{\varphi} \varphi+z_{2}^{\prime}\left(G_{\omega} u_{\omega}-S(v) \mathbf{M} v\right. \\
& -S(\omega) \mathbf{J} \omega+f_{\omega}(v, \omega, p, R)-\left[0_{3 \times 1} \mathbf{J}\right] \dot{\alpha} \\
& \left.+\left[-S(\mathbf{M} \delta)+\Gamma(\cdot)^{\prime}\right] \mathbf{M} \varphi\right) .
\end{aligned}
$$

For simplicity we did not expand the derivative of $\alpha$. If we then choose

$$
\begin{aligned}
& u_{\omega}=G_{\omega}^{-1}\left(S(v) \mathbf{M} v+S(\omega) \mathbf{J} \omega-f_{\omega}(v, \omega, p, R)+\left[0_{3 \times 1} \mathbf{J}\right] \dot{\alpha}\right. \\
& \left.-\left[-S(\mathbf{M} \delta)+\Gamma(\cdot)^{\prime}\right] \mathbf{M} \varphi-K_{z_{2}} z_{2}\right)
\end{aligned}
$$

where $K_{z_{2}} \in \mathbb{R}^{3 \times 3}$ is a symmetric positive matrix, the time derivative of $V_{3}$ becomes

$$
\dot{V}_{3}=-k_{e} e^{\prime} \mathbf{M}^{-1} e+e^{\prime} \delta-\varphi^{\prime} K_{\varphi} \varphi-z_{2}^{\prime} K_{z_{2}} z_{2} .
$$

Note that although $\dot{V}_{3}$ is not necessarily always negative, this will be sufficient to prove boundedness and convergence of $e$ to a neighborhood of the origin.

\section{B. Stability analysis}

We can now prove that all signals will remain bounded, and that the tracking error converges exponential to an arbitrarily small neighborhood of the origin.

Theorem 1: Given a sufficiently smooth time-varying desired trajectory $p_{d}:[0, \infty) \rightarrow \mathbb{R}^{3}$ with its time-derivatives bounded, consider the nonlinear system $\Sigma$ described by the underactuated vehicle model (2)-(3) in closed-loop with the feedback controller (9), (11).

i) For every initial condition of $\Sigma$, the solution exists globally, all closed-loop signals are bounded, and the tracking error $\left\|p(t)-p_{d}(t)\right\|$ satisfies

$$
\left\|p(t)-p_{d}(t)\right\| \leq e^{-\lambda t} c_{0}+\epsilon,
$$

where $\lambda, c_{0}$, and $\epsilon$ are positive constants. From these, only $c_{0}$ depends on initial conditions.

ii) For a given upper bound on $\left\|\dot{p}_{d}(t)\right\|$, by appropriate choice of the controller parameters $k_{e}, K_{\varphi}, K_{z_{2}}$, any desired values for $\epsilon$ and $\lambda$ in (12) are possible.

Proof: To prove (i) we use Young's inequality ${ }^{4}$ to conclude that for any $\gamma>0$,

$$
\begin{aligned}
\dot{V}_{3} \leq & -e^{\prime}\left(k_{e} \mathbf{M}^{-1}-\frac{\gamma}{2} I\right) e-\varphi^{\prime} K_{\varphi} \varphi \\
& -z_{2}^{\prime} K_{z_{2}} z_{2}+\frac{1}{2 \gamma}\|\delta\|^{2} .
\end{aligned}
$$

${ }^{4} \mathrm{~A}$ special case of the Young's inequality is $a b \leq \frac{\gamma}{2} a^{2}+\frac{1}{2 \gamma} b^{2}$, where $a, b \geq 0$, and $\gamma$ is any positive constant. 
Suppose now that we choose $\gamma$ sufficiently small so that the matrix $k_{e} \mathbf{M}^{-1}-\frac{\gamma}{2} I$ is positive definite. In this case we conclude that there is a sufficiently small positive constant $\lambda$ such that

$$
\dot{V}_{3} \leq-\lambda V_{3}+\frac{1}{2 \gamma}\|\delta\|^{2},
$$

and therefore it is straightforward to conclude from the Comparison Lemma [45] that

$$
V_{3}(t) \leq e^{-\lambda t} V_{3}(0)+\frac{1}{2 \lambda \gamma}\|\delta\|^{2}, \quad t \geq 0
$$

along solutions to $\Sigma$. From here we conclude that all signals remain bounded and therefore the solution exists globally. Moreover, $V_{3}$ converges to a ball of radius $\frac{1}{2 \lambda \gamma}\|\delta\|^{2}$ and therefore $\|e\|$ converges to a ball of radius $\frac{\|\delta\|}{\sqrt{\lambda \gamma}}$, because of (10).

To prove (ii), we show next that the radius $\frac{\|\delta\|}{\sqrt{\lambda \gamma}}$ can be made as small as we want by appropriately choosing the controller parameters. To this effect, suppose we pick a desired radius $\epsilon$ and a convergence rate $\lambda>0$, and we select $\delta$ such that $\mathrm{B}$ is full rank. Such value for $\delta$ may depend on the upper bound of $\left\|\dot{p}_{d}(t)\right\|$ (see Property 1 ). We can then define $\gamma:=\frac{\|\delta\|^{2}}{\epsilon^{2} \lambda}$, provided that we choose $k_{e}$ sufficiently large so that

$$
k_{e} \mathbf{M}^{-1}-\frac{\gamma}{2} I=k_{e} \mathbf{M}^{-1}-\frac{\|\delta\|^{2}}{2 \epsilon^{2} \lambda} I \geq \frac{\lambda}{2} I>0 .
$$

If we then select $K_{\varphi}:=\frac{\lambda}{2} \mathbf{M}^{2}, K_{z_{2}}:=\frac{\lambda}{2} \mathbf{J}$, we conclude from (13) that (14) indeed holds for the pre-specified $\lambda$, from which (15) follows. However, now the above choices for the parameters lead to a radius $\frac{\|\delta\|}{\sqrt{\lambda \gamma}}=\epsilon$.

Remark 2: We did not impose any constraints on the desired trajectory (besides being sufficiently smooth and its derivative being bounded) and we also did not require that the linear velocity of the vehicle be always non-null. Consequently, $p_{d}(t)$ can be arbitrary, that is, the desired trajectories do not need to satisfy "dynamic" models, and in particular can be constant for all $t \geq t_{0}$. In that case, the controller solves the position regulation problem.

Remark 3: In practice, the vector $\delta$ determines if the vehicle will follow the desired trajectory backwards or forwards. To observe this, define the following two angles: $\boldsymbol{\alpha}=\arctan \frac{e_{z}}{e_{x}}$ and $\boldsymbol{\beta}=\arctan \frac{e_{y}}{\sqrt{e_{x}^{2}+e_{z}^{2}}}$, where $e_{x}, e_{y}$, and $e_{z}$ are the three components of the body-fixed tracking error $e$. Notice that $\boldsymbol{\alpha}$ and $\boldsymbol{\beta}$ can be seen as the elevation and azimuth angles, respectively. In steady-state (with $\dot{e}=0, \dot{V}_{3}=0$ ), from (5), it follows that $e=\frac{1}{k_{e}} \mathbf{M} z_{1} \approx \frac{1}{k_{e}} \mathbf{M} \delta$. Thus, when the first component of $\delta \in \mathbb{R}^{3}$ is negative and larger (in absolute value) than the other two components, the vehicle will converge to the trajectory with positive surge velocity, and will stay "behind" the desired trajectory, see examples in Section VI.

Remark 4: When the vehicle is subject to one controlled force and only two independent control torques, i.e, $u_{v} \in \mathbb{R}$, but $u_{\omega} \in \mathbb{R}^{2}$ (and consequently $G_{\omega} \in \mathbb{R}^{3 \times 2}$ ), one can use, e.g., $V_{3}:=V_{2}+\frac{1}{2} z_{2}^{\prime} G_{\omega} P G_{\omega}^{\prime} z_{2}$, provided that there exists a symmetric positive definite matrix $P$ such that $P G_{\omega}^{\prime}=G_{\omega}^{\prime} \mathbf{J}$

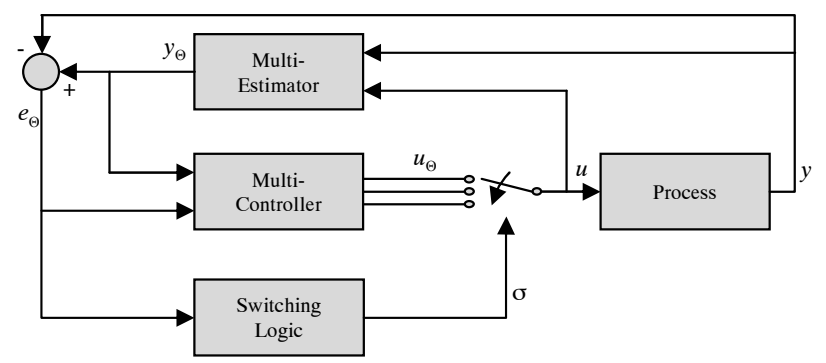

Fig. 1. Supervisory control architecture.

(which is the case for the AUV in Section VI). If we then set

$$
\begin{aligned}
u_{\omega}= & \left(G_{\omega}^{\prime} G_{\omega}\right)^{-1} G_{\omega}^{\prime}\left(S(v) \mathbf{M} v+S(\omega) \mathbf{J} \omega-f_{\omega}(v, \omega, p, R)\right. \\
& \left.+\left[0_{3 \times 1} \mathbf{J}\right] \dot{\alpha}+\left[-S(\mathbf{M} \delta)+\Gamma(\cdot)^{\prime}\right] \mathbf{M} \varphi\right)-K_{z_{2}} G_{\omega}^{\prime} z_{2}
\end{aligned}
$$

the time derivative of $V_{3}$ becomes

$$
\dot{V}_{3}=-k_{e} e^{\prime} \mathbf{M}^{-1} e+e^{\prime} \delta-\varphi^{\prime} K_{\varphi} \varphi-z_{2}^{\prime} G_{\omega} K_{z_{2}} G_{\omega}^{\prime} z_{2}+d
$$

where $d$ is a disturbance term that depends on the component of the state $z_{2}$ in the null space of $G_{\omega}^{\prime}$. From the above, one can prove boundedness if this component is bounded. For underwater vehicles this component typically corresponds to the roll motion which usually is stable due to the restoring forces.

\section{ESTIMATOR-BASED SUPERVISORY CONTROL}

Using the previous results, this section proposes an estimator-based supervisory control architecture to solve the trajectory-tracking problem in the presence of parametric modeling uncertainty. Let $\Theta \in \mathbb{R}^{n_{\ominus}}$ be a vector that contains all the unknown parameters of the dynamic equations of motion (3), where $n_{\Theta}$ denotes the number of unknown parameters. The following technical assumption is assumed to hold:

Assumption 1: Let $\mathcal{P}$ be a finite set of candidate parameter values

$$
\mathcal{P}:=\left\{\Theta_{1}, \Theta_{2}, \ldots, \Theta_{N}\right\} .
$$

The actual parameter $\Theta^{\star}$ belongs to $\mathcal{P}$.

In practice, this assumption can be relaxed to $\Theta^{\star}$ being sufficiently close to an element of $\mathcal{P}$, which can be achieved by taking a fine grid, at the price of increased computational burden. [21]:

The supervisory consists of three subsystems (see Fig. 1)

multi-estimator - a dynamical system whose inputs are the process input $u$ and its output $y$, and whose outputs are $\hat{y}_{\Theta}, \Theta \in \mathcal{P}$, where each $\hat{y}_{\Theta}$ is a suitably defined estimate of $y$ which would be asymptotically correct if $\Theta^{\star}$ was equal to $\Theta$.

multi-controller - a dynamical system whose inputs are the output estimate $\hat{y}_{\Theta}$ and the estimation errors $\mathbf{e}_{\Theta}:=\hat{y}_{\Theta}-y, \Theta \in \mathcal{P}$, and whose outputs are the control signals $u_{\Theta}, \Theta \in \mathcal{P}$, where each $u_{\Theta}$ is generated by a control law that would be adequate if $\Theta^{\star}$ was equal to $\Theta$. 
switching logic - a dynamical system whose inputs are the estimation errors $\mathbf{e}_{\Theta}$ and whose output is a switching signal $\sigma$ which is used to define the control law $u=u_{\sigma}$.

The underlying decision-making strategy used by the switching logic basically consists of selecting for $\sigma$, the candidate controller index $\Theta^{\dagger}$ for which the corresponding performance signal $\mu_{\Theta^{\dagger}}$ (which is a suitably "normed" value of $\mathbf{e}_{\Theta^{\dagger}}$ ) is currently the smallest. This strategy is motivated by the idea that the nominal process model with the smallest performance signal is the one that "best" approximates the actual process, and thus the candidate controller associated with that model can be expected to have a better performance of controlling the process.

In this paper we assume that the whole state of the process is available for feedback. Therefore, $y=(p, R, v, \omega), \hat{y}_{\Theta}=$ $\left(\hat{p}_{\Theta}, \hat{R}_{\Theta}, \hat{v}_{\Theta}, \hat{\omega}_{\Theta}\right)$, and $\mathbf{e}_{\Theta}:=\hat{y}_{\Theta}-y=\left(\tilde{v}_{\Theta}, \tilde{\omega}_{\Theta}\right)$. Since there is no uncertainty in (2), we can simply pick $\hat{p}_{\Theta}=p$ and $\hat{R}_{\Theta}=R$. We also restrict our attention to state feedback laws and therefore $u_{\Theta}=\operatorname{col}\left(u_{v_{\Theta}}, u_{\omega_{\Theta}}\right)=\mathcal{K}_{\Theta}\left(\hat{v}_{\Theta}, \hat{\omega}_{\Theta}, p, R\right)$.

\section{A. Multi-estimator}

This section addresses the design of a family of estimators parameterized by $\Theta \in \mathcal{P}$ for the underactuated vehicle model (2)-(3). Motivated by Assumption 1 and in view of (3), we consider a family of estimators of the form ${ }^{5}$

$$
\begin{aligned}
\mathbf{M}_{\Theta} \dot{\hat{v}}_{\Theta}= & -S(\omega) \mathbf{M}_{\Theta} v+f_{v_{\Theta}}(v, p, R)+g_{v_{\Theta}} u_{v}-\mathbf{M}_{\Theta} L_{v} \tilde{v}_{\Theta} \\
& -\beta_{v_{\Theta}}(\cdot) \mathbf{M}_{\Theta} \tilde{v}_{\Theta} \\
\mathbf{J}_{\Theta} \dot{\hat{\omega}}_{\Theta}= & -S(v) \mathbf{M}_{\Theta} v-S(\omega) \mathbf{J}_{\Theta} \omega+f_{\omega_{\Theta}}(v, \omega, p, R) \\
& +G_{\omega_{\Theta}} u_{\omega}-\mathbf{J}_{\Theta} L_{\omega} \tilde{\omega}_{\Theta}-\beta_{\omega_{\Theta}}(\cdot) \mathbf{J}_{\Theta} \tilde{\omega}_{\Theta}
\end{aligned}
$$

where $L_{v}, L_{\omega} \in \mathbb{R}^{3 \times 3}$ are diagonal positive definite matrices and for each $\Theta \in \mathcal{P}$ the scalar positive functions $\beta_{v_{\Theta}}\left(\hat{v}_{\Theta}, \hat{\omega}_{\Theta}, v, \omega, p, R\right):=\beta_{1_{\Theta}}(\cdot)+\beta_{2_{\Theta}}(\cdot)$ and $\beta_{\omega_{\Theta}}\left(\hat{v}_{\Theta}, \hat{\omega}_{\Theta}, \omega, p, R\right)$ satisfy $^{6}$

$$
\begin{aligned}
& \left\|f_{v_{\Theta}}\left(\hat{v}_{\Theta}, p, R\right)-f_{v_{\Theta}}(v, p, R)\right\| \\
& \quad+\left\|S(\omega) \mathbf{M}_{\Theta} \tilde{v}_{\Theta}\right\| \leq c_{1} \beta_{1_{\Theta}}\left(\hat{v}_{\Theta}, v, \omega, p, R\right)\left\|\tilde{v}_{\Theta}\right\| \\
& \left\|f_{\omega_{\Theta}}\left(\hat{v}_{\Theta}, \omega, p, R\right)-f_{\omega_{\Theta}}(v, \omega, p, R)\right\| \\
& \quad+\left\|S\left(\hat{v}_{\Theta}\right) \mathbf{M}_{\Theta}-S\left(\mathbf{M}_{\Theta} v\right)\right\|\left\|\tilde{v}_{\Theta}\right\| \\
& \quad+\left\|\left[0_{3 \times 1} \mathbf{J}_{\Theta}\right] \varphi_{v_{\Theta}}\left(\hat{v}_{\Theta}, \hat{\omega}_{\Theta}, v, \omega, p, R\right)\right\| \\
& \quad \leq c_{2} \beta_{2_{\Theta}}\left(\hat{v}_{\Theta}, \hat{\omega}_{\Theta}, v, \omega, p, R\right)\left\|\tilde{v}_{\Theta}\right\| \\
& \left\|f_{\omega_{\Theta}}\left(\hat{v}_{\Theta}, \hat{\omega}_{\Theta}, p, R\right)-f_{\omega_{\Theta}}\left(\hat{v}_{\Theta}, \omega, p, R\right)\right\| \\
& +\left\|S\left(\hat{\omega}_{\Theta}\right) \mathbf{J}_{\Theta}-S\left(\mathbf{J}_{\Theta} \omega\right)\right\|\left\|\tilde{\omega}_{\Theta}\right\| \\
& +\left\|\left[0_{3 \times 1} \mathbf{J}_{\Theta}\right] \varphi_{\omega_{\Theta}}\left(\hat{v}_{\Theta}, \hat{\omega}_{\Theta}, \omega, p, R\right)\right\| \\
& \leq c_{3} \beta_{\omega_{\Theta}}\left(\hat{v}_{\Theta}, \hat{\omega}_{\Theta}, \omega, p, R\right)\left\|\tilde{\omega}_{\Theta}\right\|
\end{aligned}
$$

for some positive constants $c_{i}, i=1, \ldots, 3$. The functions $\varphi_{v_{\Theta}}(\cdot)$ and $\varphi_{\omega_{\Theta}}(\cdot)$ will be defined later (cf. (28a)-(28b)). The multi-estimator has the desirable property that the estimator

\footnotetext{
${ }^{5}$ When $\mathcal{P}$ has a large number of elements, an alternative approach is described in Section IV-E

${ }^{6}$ The existence of $\beta_{v_{\Theta}}(\cdot)$ and $\beta_{\omega_{\Theta}}(\cdot)$ follows directly from the fact that $f_{v_{\Theta}}(\cdot)$ and $f_{\omega_{\Theta}}(\cdot)$ are $\mathcal{C}^{1}$.
}

error that corresponds to the actual parameter value $\Theta^{\star}$ converges exponentially to zero and satisfies a $\mathcal{L}_{1}$-like property.

Lemma 1: Let $\Theta^{\star} \in \mathcal{P}$ be the actual parameter value. There exist $\kappa>0, \lambda_{\mu}>0$, such that for every initial condition of (2)-(3), (16), and continuous signal $u=\operatorname{col}\left(u_{v}, u_{\omega}\right)$, there exist positive constants $\gamma_{1}, \gamma_{2}, \gamma_{3}$ that depend on the initial conditions such that

$$
\begin{gathered}
\left\|\mathbf{e}_{\Theta^{\star}}(t)\right\| \leq e^{-\kappa t} \gamma_{1} \\
\int_{0}^{t} e^{\lambda_{\mu} \tau} \beta_{v_{\Theta}}\left(\hat{v}_{\Theta^{\star}}(\tau), \hat{\omega}_{\Theta^{\star}}(\tau), v(\tau),\right. \\
\omega(\tau), p(\tau), R(\tau))\left\|\tilde{v}_{\Theta^{\star}}(\tau)\right\| d \tau \leq \gamma_{2} \\
\int_{0}^{t} e^{\lambda_{\mu} \tau} \beta_{\omega_{\Theta}}\left(\hat{v}_{\Theta^{\star}}(\tau), \hat{\omega}_{\Theta^{\star}}(\tau),\right. \\
\omega(\tau), p(\tau), R(\tau))\left\|\tilde{\omega}_{\Theta^{\star}}(\tau)\right\| d \tau \leq \gamma_{3}
\end{gathered}
$$

for every time $t$ in the maximum interval of existence of solution to the closed-loop $[0, T), T \in(0,+\infty]$.

Proof: See the Appendix.

\section{B. Multi-controller}

We now design a family of candidate feedback laws $\mathcal{K}_{\Theta}(\cdot)$ such that for each $\Theta \in \mathcal{P}, u=\operatorname{col}\left(u_{v}, u_{\omega}\right)=\mathcal{K}_{\Theta}(\cdot)$ would solve the tracking problem formulated in Section II for a process model given by (2) and (16), and "sufficiently" small estimation errors $\tilde{v}_{\Theta}, \tilde{\omega}_{\Theta}$. For a given $\Theta \in \mathcal{P}$, we design $\mathcal{K}_{\Theta}$ by constructing control-Lyapunov functions iteratively, following the design procedure proposed in Section III.

Step 1 and 2: Same as in Section III. However, in this case $z_{1}$ is re-defined as

$$
z_{1_{\Theta}}:=\hat{v}_{\Theta}-R^{\prime} \dot{p}_{d}+k_{e_{\Theta}} \mathbf{M}_{\Theta}^{-1} e
$$

and therefore $\dot{V}_{1}=-k_{e_{\Theta}} e^{\prime} \mathbf{M}_{\Theta}^{-1} e+e^{\prime} z_{1_{\Theta}}-e^{\prime} \tilde{v}_{\Theta}$.

Step 3: The dynamic equation of the error $z_{1_{\Theta}}$ is now given by

$\mathbf{M}_{\Theta} \dot{z}_{1_{\Theta}}=S\left(\mathbf{M}_{\Theta} z_{1_{\Theta}}\right) \omega+\Gamma_{\Theta}(\cdot) \omega+g_{v_{\Theta}} u_{v}+h_{1_{\Theta}}(\cdot)+h_{2_{\Theta}}(\cdot)$

where

$$
\begin{aligned}
& \Gamma_{\Theta}\left(R, \dot{p}_{d}\right):=S\left(\mathbf{M}_{\Theta} R^{\prime} \dot{p}_{d}\right)-\mathbf{M}_{\Theta} S\left(R^{\prime} \dot{p}_{d}\right) \\
& h_{1_{\Theta}}\left(e, p, R, \hat{v}_{\Theta}, z_{1_{\Theta}}, \ddot{p}_{d}\right):=f_{v_{\Theta}}\left(\hat{v}_{\Theta}, p, R\right)-\mathbf{M}_{\Theta} R^{\prime} \ddot{p}_{d} \\
& +k_{e_{\Theta}} z_{1_{\Theta}}-k_{e_{\Theta}}^{2} \mathbf{M}_{\Theta}^{-1} e \\
& h_{2_{\Theta}}\left(\tilde{v}_{\Theta}, \hat{v}_{\Theta}, v, \omega, p, R\right):=-\mathbf{M}_{\Theta} L_{v} \tilde{v}_{\Theta}-k_{e_{\Theta}} \tilde{v}_{\Theta} \\
& -\beta_{v_{\Theta}}\left(\hat{v}_{\Theta}, \hat{\omega}_{\Theta}, v, \omega, p, R\right) \mathbf{M}_{\Theta} \tilde{v}_{\Theta}+S(\omega) \mathbf{M}_{\Theta} \tilde{v}_{\Theta} \\
& +f_{v_{\Theta}}(v, p, R)-f_{v_{\Theta}}\left(\hat{v}_{\Theta}, p, R\right)
\end{aligned}
$$

Thus, $V_{2}$ is re-defined as

$$
V_{2}:=V_{1}+\frac{1}{2} \varphi_{\Theta}^{\prime} \mathbf{M}_{\Theta}^{2} \varphi_{\Theta}=\frac{1}{2} e^{\prime} e+\frac{1}{2} \varphi_{\Theta}^{\prime} \mathbf{M}_{\Theta}^{2} \varphi_{\Theta}
$$

where $\varphi_{\Theta}:=z_{1_{\Theta}}-\delta_{\Theta}$. The time derivative of $V_{2}$ can be written as

$$
\begin{aligned}
\dot{V}_{2}= & -k_{e_{\Theta}} e^{\prime} \mathbf{M}_{\Theta}^{-1} e+e^{\prime} \delta_{\Theta}-e^{\prime} \tilde{v}_{\Theta} \\
& +\varphi_{\Theta}^{\prime}\left[\mathbf{M}_{\Theta} \mathrm{B}_{\Theta}(\cdot) \zeta_{\Theta}+\mathbf{M}_{\Theta} h_{1_{\Theta}}(\cdot)+h_{3_{\Theta}}(\cdot)+e\right]
\end{aligned}
$$


where

$$
\begin{aligned}
& h_{3_{\Theta}}\left(\tilde{v}_{\Theta}, \tilde{\omega}_{\Theta}, \hat{v}_{\Theta}, \dot{p}_{d}, v, \omega, p, R\right):=\mathbf{M}_{\Theta} h_{2_{\Theta}}(\cdot) \\
& \quad-\mathbf{M}_{\Theta}\left[S\left(\mathbf{M}_{\Theta} \delta_{\Theta}\right)+\Gamma_{\Theta}(\cdot)\right] \tilde{\omega}_{\Theta} \\
& \mathrm{B}_{\Theta}\left(R, \dot{p}_{d}, \delta_{\Theta}\right):=\left[\begin{array}{ll}
g_{v_{\Theta}} & S\left(\mathbf{M}_{\Theta} \delta_{\Theta}\right)+\Gamma_{\Theta}\left(R, \dot{p}_{d}\right)
\end{array}\right] \\
& \zeta_{\Theta}:=\operatorname{col}\left(u_{v}, \hat{\omega}_{\Theta}\right) .
\end{aligned}
$$

Following the same line of reason described in Step 3 of Section III, let

$$
\alpha_{\Theta}:=\mathrm{B}_{\Theta}^{\prime}\left(\mathrm{B}_{\Theta} \mathrm{B}_{\Theta}^{\prime}\right)^{-1}\left(-h_{1_{\Theta}}(\cdot)-\mathbf{M}_{\Theta}^{-1} e-\mathbf{M}_{\Theta}^{-1} K_{\varphi_{\Theta}} \varphi_{\Theta}\right)
$$

be a virtual control law for each $\Theta \in \mathcal{P}$, where $\delta_{\Theta}$ is chosen such that $\mathrm{B}_{\Theta} \mathrm{B}_{\Theta}^{\prime}$ is nonsingular. Let $u_{v}$ be equal to the first entry of $\alpha_{\Theta}$, i.e.,

$$
u_{1}=\left[\begin{array}{ll}
1 & 0_{1 \times 3}
\end{array}\right] \alpha_{\Theta}
$$

and

$$
z_{2 \Theta}:=\hat{\omega}_{\Theta}-\left[\begin{array}{ll}
0_{3 \times 1} & I_{3 \times 3}
\end{array}\right] \alpha_{\Theta} .
$$

Then,

$$
\begin{aligned}
\dot{V}_{2}= & -k_{e_{\Theta}} e^{\prime} \mathbf{M}_{\Theta}^{-1} e+e^{\prime} \delta_{\Theta}-e^{\prime} \tilde{v}_{\Theta}-\varphi_{\Theta}^{\prime} K_{\varphi_{\Theta}} \varphi_{\Theta} \\
& +\varphi_{\Theta}^{\prime} \mathbf{M}_{\Theta}\left[S\left(M_{\Theta} \delta_{\Theta}\right)+\Gamma_{\Theta}(\cdot)\right] z_{2_{\Theta}}+\varphi_{\Theta}^{\prime} h_{3_{\Theta}}(\cdot) .
\end{aligned}
$$
by

Step 4: The third control-Lyapunov function is now given

$$
V_{3}:==\frac{1}{2} e^{\prime} e+\frac{1}{2} \varphi_{\Theta}^{\prime} \mathbf{M}_{\Theta}^{2} \varphi_{\Theta}+\frac{1}{2} z_{2_{\Theta}}^{\prime} \mathbf{J}_{\Theta} z_{2_{\Theta}} .
$$

Computing its time derivative one obtains

$$
\begin{aligned}
\dot{V}_{3}= & -k_{e_{\Theta}} e^{\prime} \mathbf{M}_{\Theta}^{-1} e+e^{\prime} \delta_{\Theta}-e^{\prime} \tilde{v}_{\Theta}-\varphi_{\Theta}^{\prime} K_{\varphi_{\Theta}} \varphi_{\Theta} \\
& +\varphi_{\Theta}^{\prime} h_{3 \Theta}(\cdot)+z_{2_{\Theta}}^{\prime}\left[G_{\omega_{\Theta}} u_{\omega}-S(v) \mathbf{M}_{\Theta} v-S(\omega) \mathbf{J}_{\Theta} \omega\right. \\
& +f_{\omega_{\Theta}}(v, \omega, p, R)-\mathbf{J}_{\Theta} L_{\omega} \tilde{\omega}_{\Theta}-\beta_{\omega_{\Theta}}(\cdot) \mathbf{J}_{\Theta} \tilde{\omega}_{\Theta} \\
& \left.-\left[0_{3 \times 1} \mathbf{J}_{\Theta}\right] \dot{\alpha}_{\Theta}+\left[-S\left(M_{\Theta} \delta_{\Theta}\right)+\Gamma_{\Theta}(\cdot)^{\prime}\right] \mathbf{M}_{\Theta} \varphi_{\Theta}\right]
\end{aligned}
$$

where $\dot{\alpha}_{\Theta}$ can be decomposed in two terms: $\dot{\alpha}_{\Theta}=\dot{\hat{\alpha}}_{\Theta}-\dot{\tilde{\alpha}}_{\Theta}$. Here, $\dot{\tilde{\alpha}}_{\Theta}:=\dot{\hat{\alpha}}_{\Theta}-\dot{\alpha}_{\Theta}$, and $\dot{\hat{\alpha}}_{\Theta}$ is defined to be the same as $\dot{\alpha}_{\Theta}$, but substituting the arguments $v, \omega$ by $\hat{v}_{\Theta}, \hat{\omega}_{\Theta}$, respectively. Selecting

$$
\begin{aligned}
u_{\omega}= & G_{\omega_{\Theta}}^{-1}\left(S\left(\hat{v}_{\Theta}\right) \mathbf{M}_{\Theta} \hat{v}_{\Theta}+S\left(\hat{\omega}_{\Theta}\right) \mathbf{J}_{\Theta} \hat{\omega}_{\Theta}\right. \\
& -f_{\omega_{\Theta}}\left(\hat{v}_{\Theta}, \hat{\omega}_{\Theta}, p, R\right)+\left[0_{3 \times 1} \mathbf{J}_{\Theta}\right] \dot{\hat{\alpha}}_{\Theta}-\left[-S\left(M_{\Theta} \delta_{\Theta}\right)\right. \\
& \left.\left.+\Gamma_{\Theta}(\cdot)^{\prime}\right] \mathbf{M}_{\Theta} \varphi_{\Theta}-K_{z_{z}} z_{2_{\Theta}}\right)
\end{aligned}
$$

where for each $\Theta \in \mathcal{P}, K_{z_{2_{\Theta}}} \in \mathbb{R}^{3 \times 3}$ is a symmetric positive matrix, the time derivative of $V_{3}$ becomes

$$
\begin{aligned}
\dot{V}_{3}= & -k_{e_{\Theta}} e^{\prime} \mathbf{M}_{\Theta}^{-1} e-\varphi_{\Theta}^{\prime} K_{\varphi_{\Theta}} \varphi_{\Theta}-z_{2_{\Theta}}^{\prime} K_{z_{2_{\Theta}}} z_{2_{\Theta}}+e^{\prime} \delta_{\Theta} \\
& -e^{\prime} \tilde{v}_{\Theta}+\varphi_{\Theta}^{\prime} h_{3 \Theta}(\cdot)+z_{2_{\Theta}}^{\prime} h_{4_{\Theta}}(\cdot)
\end{aligned}
$$

where

$$
\begin{aligned}
h_{4_{\Theta}} & \left.\hat{v}_{\Theta}, \hat{\omega}_{\Theta}, v, \omega, p, R\right):=S\left(\hat{v}_{\Theta}\right) \mathbf{M}_{\Theta} \hat{v}_{\Theta}-S(v) \mathbf{M}_{\Theta} v \\
& +S\left(\hat{\omega}_{\Theta}\right) \mathbf{J}_{\Theta} \hat{\omega}_{\Theta}-S(\omega) \mathbf{J}_{\Theta} \omega+f_{\omega_{\Theta}}(v, \omega, p, R) \\
& -f_{\omega_{\Theta}}\left(\hat{v}_{\Theta}, \hat{\omega}_{\Theta}, p, R\right)-\mathbf{J}_{\Theta} L_{\omega} \tilde{\omega}_{\Theta}-\beta_{\omega_{\Theta}}(\cdot) \mathbf{J}_{\Theta} \tilde{\omega}_{\Theta} \\
& -\left[0_{3 \times 1} \mathbf{J}_{\Theta}\right]\left(\dot{\alpha}_{\Theta}-\dot{\hat{\alpha}}_{\Theta}\right)
\end{aligned}
$$

The last term $\dot{\alpha}_{\Theta}-\dot{\hat{\alpha}}_{\Theta}$ can be rewritten as $\dot{\alpha}_{\Theta}-\dot{\hat{\alpha}}_{\Theta}=\varphi_{v_{\Theta}}+$ $\varphi_{\omega_{\Theta}}$, where

$$
\begin{aligned}
\varphi_{v_{\Theta}}\left(\hat{v}_{\Theta}, \hat{\omega}_{\Theta}, v, \omega, p, R\right):= & \dot{\alpha}_{\Theta}\left(\hat{v}_{\Theta}, \hat{\omega}_{\Theta}, v, \omega, p, R\right) \\
& -\dot{\alpha}_{\Theta}\left(\hat{v}_{\Theta}, \hat{\omega}_{\Theta}, \hat{v}_{\Theta}, \omega, p, R\right) \\
\varphi_{\omega_{\Theta}}\left(\hat{v}_{\Theta}, \hat{\omega}_{\Theta}, \omega, p, R\right):= & \dot{\alpha}_{\Theta}\left(\hat{v}_{\Theta}, \hat{\omega}_{\Theta}, \hat{v}_{\Theta}, \omega, p, R\right) \\
& -\dot{\alpha}_{\Theta}\left(\hat{v}_{\Theta}, \hat{\omega}_{\Theta}, \hat{v}_{\Theta}, \hat{\omega}_{\Theta}, p, R\right)
\end{aligned}
$$

From (26), although $\dot{V}_{3}$ has indefinite terms, it will be verified that they will be dominated by the negative definite terms when the estimator errors $\tilde{v}_{\Theta}, \tilde{\omega}_{\Theta}$ are sufficiently small. This is stated in the following lemma.

Lemma 2: Let $[0, T), T \in[0, \infty]$ denote the maximum interval of existence of solution to the closed-loop and suppose that there exists a time $T^{\dagger} \geq 0$ such that $u(t)=\mathcal{K}_{\Theta^{\dagger}}(\cdot)$ for all $t \in\left[T^{\dagger}, T\right)$ and

$$
\int_{T^{\dagger}}^{T} \gamma\left(y(\tau), \tilde{v}_{\Theta^{\dagger}}(\tau), \tilde{\omega}_{\Theta^{\dagger}}(\tau)\right) d \tau<\infty
$$

where the control law $\mathcal{K}_{\Theta}$ is defined in (22) and (25) and

$$
\begin{aligned}
\gamma(\cdot):= & \left\|\mathbf{e}_{\Theta^{\dagger}}\right\|^{2}+\beta_{v_{\Theta}}\left(v+\tilde{v}_{\Theta^{\dagger}}, \omega+\tilde{\omega}_{\Theta^{\dagger}}, v, w, p, R\right)\left\|\tilde{v}_{\Theta^{\dagger}}\right\| \\
& +\beta_{\omega_{\Theta}}\left(v+\tilde{v}_{\Theta^{\dagger}}, w+\tilde{\omega}_{\Theta^{\dagger}}, w, p, R\right)\left\|\tilde{\omega}_{\Theta^{\dagger}}\right\| .
\end{aligned}
$$

Given a sufficiently smooth time-varying desired trajectory $p_{d}:[0, T) \rightarrow \mathbb{R}^{3}$ with its time-derivatives bounded and any initial condition of the resulting closed-loop system, the signals $e(t), \hat{v}_{\Theta}(t), \hat{\omega}_{\Theta}(t)$, and $u(t)$ are bounded on $\left[T^{\dagger}, T\right)$. Moreover, if (29) holds with $T=+\infty$, then, as $t \rightarrow \infty$, the tracking error $\left\|p(t)-p_{d}(t)\right\|$ converges to a neighborhood of the origin that can be made arbitrarily small by appropriate choice of the controller parameters.

Proof: See the Appendix.

Loosely speaking, Lemma 2 states that each candidate controller solves the trajectory-tracking problem formulated in Section II provided that the input disturbances due to the estimation errors have finite energy as defined by the integral (29). The switching-logic will guarantee that (29) holds by the Scale-Independent Hysteresis Switching Lemma [21] (cf. proof of Theorem 2).

\section{Switching-logic}

Motivated by (29)-(30), for each $\Theta \in \mathcal{P}$, we start by defining the performance signal $\mu_{\Theta}$ as the state of the dynamic equation

$$
\dot{\mu}_{\Theta}=-\lambda_{\mu} \mu_{\Theta}+\gamma\left(y, \tilde{v}_{\Theta}, \tilde{\omega}_{\Theta}\right),
$$

with the initial values satisfying $\mu_{\Theta}(0)>0$. Equation (31) implies that each performance signal $\mu_{\Theta}$ is the sum of an exponentially decaying term that depends on initial conditions and a suitable exponentially weighted "norm" of the corresponding estimation errors. The control parameter $\lambda_{\mu}$ acts as a forgetting factor in the evaluation of the performance signals, hence establishing a compromise between adaptation alertness and switching dither.

The switching logic consider here is the scale-independent hysteresis switching logic proposed in [21]. Let $h$ be a positive constant called the hysteresis constant. The operation of the 
switching logic can be briefly explained as follows: First, we set $\sigma(0)=\arg \min _{\Theta \in \mathcal{P}}\left\{\mu_{\Theta}(0)\right\}$. Suppose that at a certain time $t_{i}$ the value of $\sigma$ has just switched to some $\Theta^{\dagger} \in \mathcal{P}$. Then, $\sigma$ will be kept fixed until a time $t_{i+1}>t_{i}$ such that $(1+h) \min _{\Theta \in \mathcal{P}}\left\{\mu_{\Theta}\left(t_{i+1}\right)\right\} \leq \mu_{\Theta^{\dagger}}\left(t_{i+1}\right)$, at which point we set $\sigma\left(t_{i+1}\right)$ to $\sigma\left(t_{i+1}\right)=\arg \min _{\Theta \in \mathcal{P}}\left\{\mu_{\Theta}\left(t_{i+1}\right)\right\}$. When the indicated minimum is not unique, a particular value for $\sigma$ among those that achieve the minimum can be chosen arbitrarily. Repeating this procedure, a piecewise constant signal $\sigma$ is generated that is continuous from right everywhere. Setting $\mu_{\Theta}(0)>0$ for all $\Theta \in \mathcal{P}$ avoids chattering. The switching signal is used to define the control signal as follows:

$$
u=\operatorname{col}\left(u_{v}, u_{\omega}\right)=\mathcal{K}_{\sigma}\left(\hat{v}_{\Theta}, \hat{\omega}_{\Theta}, p, R\right),
$$

where the candidate control laws $\mathcal{K}_{\Theta}$ are defined by (22), (25).

\section{Stability analysis}

We are now ready to prove that all closed-loop signals will remain bounded, and that the tracking error converges to an arbitrarily small neighborhood of the origin in the presence of possible large parametric modeling uncertainty.

Theorem 2: Given a sufficiently smooth time-varying desired trajectory $p_{d}:[0, \infty) \rightarrow \mathbb{R}^{3}$ with its time-derivatives bounded, consider the hybrid system $\Sigma_{\text {track }}$ described by the underactuated vehicle model (2)-(3) in closed-loop with the switched multi-controller (32), the multi-estimator (16), and the switching logic described in Section IV-C.

i) For any initial condition of $\Sigma_{\text {track }}$ with $\mu_{\Theta}(0)>0, \forall \Theta \in$ $\mathcal{P}$, the solution exists globally and all closed-loop signals are bounded.

ii) Furthermore, there exists a finite time $T^{\dagger} \geq 0$ such that $\sigma(t)=\Theta^{\dagger} \in \mathcal{P}$ for all $t \geq T^{\dagger}$ (i.e., the switching stops in finite time) and as $t \rightarrow \infty$ the tracking error $\| p(t)-$ $p_{d}(t) \|$ converges to a neighborhood of the origin that can be made arbitrarily small by appropriate choice of the control parameters.

Proof: Consider the scaled performance signals $\bar{\mu}_{\Theta}(t):=$ $e^{\lambda_{\mu} t} \mu_{\Theta}(t), \Theta \in \mathcal{P}$. From (31) we conclude that

$\bar{\mu}_{\Theta}(t)=\bar{\mu}_{\Theta}(0)+\int_{0}^{t} e^{\lambda_{\mu} \tau} \gamma\left(y(\tau), \tilde{v}_{\Theta}(\tau), \tilde{\omega}_{\Theta}(\tau)\right) d \tau, \Theta \in \mathcal{P}$.

Because of the scale independence property of the switching logic, replacing $\mu_{\Theta}$ by $\bar{\mu}_{\Theta}$ would have no effect on $\sigma$. From (33) we see that each $\bar{\mu}_{\Theta}$ is nondecreasing. This, the finiteness of $\mathcal{P}$, and the fact that $\bar{\mu}_{\Theta}(0)>0$ for each $\Theta \in \mathcal{P}$ guarantee the existence of a positive number $\epsilon$ such that $\bar{\mu}_{\Theta}(t)>\epsilon, \forall t \geq$ $0, \forall \Theta \in \mathcal{P}$. It is not hard to conclude from the definition of the switching logic that chattering cannot occur. In fact, there must be an interval $[0, T)$ of maximal length on which the solution of the system is defined, and $\sigma$ can only have a finite number of discontinuities on each proper subinterval of $[0, T)$. For details, see [21]. To prove that the switching stops in finite time, observe from (33) and (30) that $\bar{\mu}_{\Theta^{\star}}$ is bounded by virtue of Lemma 1 . It follows now that the signals $\bar{\mu}_{\Theta}$ satisfy the hypotheses of the Scale-Independent Hysteresis Switching Lemma [21] which enables us to conclude that the switching stops in finite time. More precisely, there exists a time $T^{\dagger}<T$ such that $\sigma(t)=\Theta^{\dagger} \in \mathcal{P}$ for all $t \in\left[T^{\dagger}, T\right)$. In addition, $\bar{\mu}_{\Theta^{\dagger}}$ is bounded on $[0, T)$. Using (33) with $\Theta=\Theta^{\dagger}$ and the boundedness of $\bar{\mu}_{\Theta^{\dagger}}$, we see that the integral

$$
\int_{T^{\dagger}}^{T} \gamma\left(y(\tau), \tilde{v}_{\Theta^{\dagger}}(\tau), \tilde{\omega}_{\Theta^{\dagger}}(\tau)\right) d \tau
$$

is finite (recall that $\lambda_{\mu}$ is positive). Therefore, resorting to Lemma 2 , this implies that $\hat{v}_{\Theta^{\dagger}}, \hat{\omega}_{\Theta^{\dagger}}, e$, and $u$ are bounded on $\left[T^{\dagger}, T\right)$.

Next we will prove that $\hat{v}_{\Theta^{\star}}$ and $\hat{\omega}_{\Theta^{\star}}$ are also bounded on $\left[T^{\dagger}, T\right)$, where $\Theta^{\star} \in \mathcal{P}$ is the actual parameter value. Consider the following nonnegative function

$$
V=\frac{1}{2} \hat{v}_{\Theta^{\star}}^{\prime} \mathbf{M} \hat{v}_{\Theta^{\star}}+\frac{1}{2} \hat{\omega}_{\Theta^{\star}}^{\prime} \mathbf{J} \hat{\omega}_{\Theta^{\star}} .
$$

Its time derivative satisfies (c.f. Appendix)

$$
\dot{V} \leq-\lambda V+\left[a_{1}(t)+a_{2}(t)\right] V+b_{1}(t)+b_{2}(t)+\kappa_{1},
$$

where $\lambda>0, a_{1}(t), b_{1}(t)$ are functions in $\mathcal{L}_{1}$ defined on $\left[T^{\dagger}, T\right)$, and $a_{2}(t), b_{2}(t)$ are functions in $\mathcal{L}_{2}$ defined on $\left[T^{\dagger}, T\right)$. Consider now the ordinary differential equation

$$
\begin{aligned}
\dot{x}(t)= & -\lambda x(t)+\left[a_{1}(t)+a_{2}(t)\right] x(t) \\
& +b_{1}(t)+b_{2}(t)+\kappa_{1}, \quad t \in\left[T^{\dagger}, T\right)
\end{aligned}
$$

Using [46, Lemma 1] we conclude that for any initial condition, the solution $x(t)$ to (36) exists and is bounded on $\left[T^{\dagger}, T\right)$. Moreover, when $T=+\infty, x(t)$ converges to $\frac{\kappa_{1}}{\lambda}$ as $t \rightarrow+\infty$. Thus, applying the Comparison Lemma to (35) it can be concluded that $V(t)$ and, consequently, $\hat{v}_{\Theta^{\star}}, \hat{\omega}_{\Theta^{*}}$ are bounded on $\left[T^{\dagger}, T\right)$. Since $\mathbf{e}_{\Theta^{*}}$ is bounded by virtue of Lemma 1 , it follows that $\left(v^{\prime}, \omega^{\prime}\right)=\left(\hat{v}_{\Theta^{\star}}^{\prime}, \hat{\omega}_{\Theta^{\star}}^{\prime}\right)-\mathbf{e}_{\Theta^{\star}}^{\prime}$ is bounded on $\left[T^{\dagger}, T\right)$. Combining the boundedness of $(v, \omega)$ and $\mathcal{K}_{\Theta^{\dagger}}$ with the estimators (16), it can be seen that the dynamic equations for the quadratic estimation error $\left\|\mathbf{e}_{\Theta}\right\|^{2}, \Theta \in \mathcal{P}$ can be expressed (after applying the Comparison Lemma) as an exponential stable linear system with bounded inputs. Therefore, this implies that $\mathbf{e}_{\Theta}$ and $\left(\hat{v}_{\Theta}^{\prime}, \hat{\omega}_{\Theta}^{\prime}\right)=\left(v^{\prime}, \omega^{\prime}\right)+\mathbf{e}_{\Theta}^{\prime}$ are bounded on $\left[T^{\dagger}, T\right)$ for each $\Theta \in \mathcal{P}$. Since all signals are bounded in the maximal interval of existence of solutions, one conclude that the solutions exist globally, i.e., $T=+\infty$. The convergence of the tracking error $\left\|p(t)-p_{d}(t)\right\|$ to a neighborhood of the origin now follows from Lemma 2.

\section{E. State-sharing}

In the previous sections we have relied on the fact that the set $\mathcal{P}$ was finite, so that the estimators (16). If the set $\mathcal{P}$ is infinite or it has a large number of elements, a different approach is required. One alternative, which leads to a more efficient design, is to replace the individual estimator equations by a single system and use it to generate the estimation errors. In other words, to make the estimators in (16) "share" the same state. The performance signals $\mu_{\Theta}$ can be obtained in a similar way. To this effect, suppose that the functions $f_{v_{\Theta}}(v, p, R)$, and $f_{\omega_{\Theta}}(v, \omega, p, R)$ are separable on the unknown parameter $\Theta$, i.e, take the form $a^{\prime}(v, \omega, p, R) b(\Theta)$. In that case, we can 
define

$$
\begin{aligned}
a_{v}^{\prime}\left(v, \omega, p, R, u_{v}\right) b_{v}(\Theta):= & -\mathbf{M}_{\Theta}^{-1} S(\omega) \mathbf{M}_{\Theta} v \\
& +\mathbf{M}_{\Theta}^{-1} f_{v_{\Theta}}(v, p, R)+\mathbf{M}_{\Theta}^{-1} g_{v_{\Theta}} u_{v} \\
a_{\omega}^{\prime}\left(v, \omega, p, R, u_{\omega}\right) b_{\omega}(\Theta):= & -\mathbf{J}_{\Theta}^{-1} S(v) \mathbf{M}_{\Theta} v-\mathbf{J}_{\Theta}^{-1} S(\omega) \mathbf{J}_{\Theta} \omega \\
& +\mathbf{J}_{\Theta}^{-1} f_{\omega_{\Theta}}(v, \omega, p, R)+\mathbf{J}_{\Theta}^{-1} G_{\omega \Theta} u_{\omega}
\end{aligned}
$$

and replace the estimators (16) by

$$
\begin{aligned}
\dot{\hat{x}}_{v_{1}}= & -L_{v} \hat{x}_{v_{1}}-\bar{\beta}_{v}\left(\hat{x}_{v}, v, \omega, p, R\right) \hat{x}_{v_{1}}+L_{v} v \\
& +\bar{\beta}_{v}\left(\hat{x}_{v}, v, \omega, p, R\right) v \\
\dot{\hat{x}}_{v_{2}}= & -L_{v} \hat{x}_{v_{2}}-\bar{\beta}_{v}\left(\hat{x}_{v}, v, \omega, p, R\right) \hat{x}_{v_{2}} \\
& +a_{v}^{\prime}\left(v, \omega, p, R, u_{v}\right) \\
\dot{\hat{x}}_{\omega_{1}}= & -L_{\omega} \hat{x}_{\omega_{1}}-\bar{\beta}_{\omega}\left(x_{v}, x_{\omega}, \omega, p, R\right) \hat{x}_{\omega_{1}}+L_{\omega} \omega \\
& +\bar{\beta}_{\omega}\left(x_{v}, x_{\omega}, \omega, p, R\right) \omega \\
\dot{\hat{x}}_{\omega_{2}}= & -L_{\omega} \hat{x}_{\omega_{2}}-\bar{\beta}_{\omega}\left(x_{v}, x_{\omega}, \omega, p, R\right) \hat{x}_{\omega_{2}} \\
& +a_{\omega}^{\prime}\left(v, \omega, p, R, u_{\omega}\right)
\end{aligned}
$$

with outputs

$$
\hat{v}_{\Theta}=\hat{x}_{v_{1}}+\hat{x}_{v_{2}} b_{v}(\Theta), \quad \hat{\omega}_{\Theta}=\hat{x}_{\omega_{1}}+\hat{x}_{\omega_{2}} b_{\omega}(\Theta)
$$

where

$$
\begin{array}{r}
\bar{\beta}_{v}\left(\hat{x}_{v}, v, \omega, p, R\right)=\sup _{\Theta} \beta_{v_{\Theta}}\left(\hat{x}_{v_{1}}+\hat{x}_{v_{2}} b_{v}(\Theta), \hat{x}_{\omega_{1}}\right. \\
\left.+\hat{x}_{\omega_{2}} b_{\omega}(\Theta), v, \omega, p, R\right) \\
\bar{\beta}_{\omega}\left(x_{v}, x_{\omega}, \omega, p, R\right)=\sup _{\Theta} \beta_{\omega_{\Theta}}\left(\hat{x}_{v_{1}}+\hat{x}_{v_{2}} b_{v}(\Theta), \hat{x}_{\omega_{1}}\right. \\
\left.+\hat{x}_{\omega_{2}} b_{\omega}(\Theta), \omega, p, R\right) .
\end{array}
$$

Note that $\hat{v}_{\Theta}$ and $\hat{\omega}_{\Theta}$ satisfy (16) but with possible larger $\beta_{v_{\Theta}}$ and $\beta_{\omega_{\Theta}}$ that still satisfy (17). However, the dimension of (37) is now independent of the number of elements in $\mathcal{P}$.

\section{PATh-Following CONTROLler Design}

In this section, inspired by [39], the results described in Section IV are utilized to solve the path-following problem. Let $p_{d}(\gamma) \in \mathbb{R}^{3}$ be a desired geometric path parameterized by a variable $\gamma \in \mathbb{R}$ and $v_{r}(\gamma) \in \mathbb{R}$ a desired speed assignment. Contrary to trajectory-tracking, in path-following we have the freedom to select a timing law for $\gamma(t)$. In particular, we can regard $\gamma(t)$ as an additional control input. In this paper, we actually regard $\ddot{\gamma}(t)$ as the additional input, because this will necessarily produce a differentiable $\gamma(t)$. Let us define the position body-fixed path-following error $e:=R^{\prime}[p(t)-$ $\left.p_{d}(\gamma(t))\right]$ and the speed error $z_{\gamma}:=\dot{\gamma}-v_{r}$. Following the same steps described in Section III and IV-B, and defining $z_{1_{\Theta}}$ (see (19)) as $z_{1_{\Theta}}:=\hat{v}_{\Theta}-R^{\prime} p_{d}^{\gamma} v_{r}+k_{e_{\Theta}} \mathbf{M}_{\Theta}^{-1} e$, where $p_{d}^{\gamma}:=\frac{\partial p_{d}}{\partial \gamma}$, we obtain

$$
\dot{V}_{1}=-k_{e_{\Theta}} e^{\prime} \mathbf{M}_{\Theta}^{-1} e+e^{\prime} z_{1_{\Theta}}-e^{\prime} \tilde{v}_{\Theta}-e^{\prime} R^{\prime} p_{d}^{\gamma} z_{\gamma} .
$$

Notice also that

$$
\begin{aligned}
\mathbf{M}_{\Theta} \dot{z}_{1_{\Theta}}= & S\left(\mathbf{M}_{\Theta} z_{1_{\Theta}}\right) \omega+\Gamma_{\Theta}(\cdot) \omega+g_{v_{\Theta}} u_{v} \\
& +h_{1_{\Theta}}(\cdot)+h_{2_{\Theta}}(\cdot)+h_{5_{\Theta}}(\cdot) z_{\gamma}
\end{aligned}
$$

where

$\Gamma_{\Theta}\left(R, p_{d}^{\gamma}, v_{r}\right):=S\left(\mathbf{M}_{\Theta} R^{\prime} p_{d}^{\gamma} v_{r}\right)-\mathbf{M}_{\Theta} S\left(R^{\prime} p_{d}^{\gamma} v_{r}\right)$

$$
\begin{aligned}
& h_{1_{\Theta}}\left(e, p, R, \hat{v}_{\Theta}, z_{1_{\Theta}}, p_{d}^{\gamma}, p_{d}^{\gamma^{2}}, v_{r}, v_{r}^{\gamma}\right):=f_{v_{\Theta}}\left(\hat{v}_{\Theta}, p, R\right) \\
& \quad-\mathbf{M}_{\Theta} R^{\prime}\left(p_{d}^{\gamma^{2}} v_{r}^{2}+p_{d}^{\gamma} v_{r}^{\gamma} v_{r}\right)+k_{e_{\Theta}} z_{1_{\Theta}}-k_{e_{\Theta}}^{2} \mathbf{M}_{\Theta}^{-1} e \\
& h_{2_{\Theta}}\left(\tilde{v}_{\Theta}, \hat{v}_{\Theta}, v, \omega, p, R\right):=-\mathbf{M}_{\Theta} L_{v} \tilde{v}_{\Theta} \\
& \quad-\beta_{v_{\Theta}}\left(\hat{v}_{\Theta}, \hat{\omega}_{\Theta}, v, \omega, p, R\right) \mathbf{M}_{\Theta} \tilde{v}_{\Theta}-k_{e_{\Theta}} \tilde{v}_{\Theta}+S(\omega) \mathbf{M}_{\Theta} \tilde{v}_{\Theta} \\
& \quad+f_{v_{\Theta}}(v, p, R)-f_{v_{\Theta}}\left(\hat{v}_{\Theta}, p, R\right)
\end{aligned}
$$$$
h_{5_{\Theta}}\left(R, p_{d}^{\gamma}, p_{d}^{\gamma^{2}}, v_{r}, v_{r}^{\gamma}\right):=-M_{\Theta} R^{\prime}\left(p_{d}^{\gamma^{2}} v_{r}+p_{d}^{\gamma} v_{r}^{\gamma}\right)-k_{e_{\Theta}} R^{\prime} p_{d}^{\gamma}
$$

and $p_{d}^{\gamma^{2}}:=\frac{\partial^{2} p_{d}}{\partial \gamma^{2}}, v_{r}^{\gamma}:=\frac{\partial v_{r}}{\partial \gamma}$. Therefore, using (21)-(23), we obtain

$$
\begin{aligned}
\dot{V}_{2}= & -k_{e_{\Theta}} e^{\prime} \mathbf{M}_{\Theta}^{-1} e+e^{\prime} \delta_{\Theta}-e^{\prime} \tilde{v}_{\Theta}-\varphi_{\Theta}^{\prime} K_{\varphi_{\Theta}} \varphi_{\Theta} \\
& +\varphi_{\Theta}^{\prime} \mathbf{M}_{\Theta}\left[S\left(M_{\Theta} \delta_{\Theta}\right)+\Gamma_{\Theta}\right] z_{2_{\Theta}} \\
& +\varphi_{\Theta}^{\prime} h_{3_{\Theta}}(\cdot)+\left[-e^{\prime} R^{\prime} p_{d}^{\gamma}+\varphi_{\Theta}^{\prime} \mathbf{M}_{\Theta} h_{5_{\Theta}}(\cdot)\right] z_{\gamma}
\end{aligned}
$$

where

$$
\begin{aligned}
& h_{3_{\Theta}}\left(\tilde{v}_{\Theta}, \tilde{\omega}_{\Theta}, \hat{v}_{\Theta}, p_{d}^{\gamma}, v_{r}, v, \omega, p, R\right):=\mathbf{M}_{\Theta} h_{2 \Theta}(\cdot) \\
& \quad-\mathbf{M}_{\Theta}\left[S\left(\mathbf{M}_{\Theta} \delta_{\Theta}\right)+\Gamma_{\Theta}(\cdot)\right] \tilde{\omega}_{\Theta} \\
& \mathrm{B}_{\Theta}\left(R, p_{d}^{\gamma}, v_{r}, \delta_{\Theta}\right):=\left[g_{v_{\Theta}}, S\left(\mathbf{M}_{\Theta} \delta_{\Theta}\right)+\Gamma_{\Theta}\left(R, p_{d}^{\gamma}, v_{r}\right)\right] \\
& \zeta_{\Theta}:=\operatorname{col}\left(u_{v}, \hat{\omega}_{\Theta}\right)
\end{aligned}
$$

Notice that $\dot{\alpha}_{\Theta}$ can be decomposed as $\dot{\alpha}_{\Theta}=h_{6_{\Theta}}(\cdot)+h_{7 \Theta}(\cdot) z_{\gamma}$. Thus, if we then choose

$$
\begin{aligned}
u_{\omega}= & G_{\omega_{\Theta}}^{-1}\left(S\left(\hat{v}_{\Theta}\right) \mathbf{M}_{\Theta} \hat{v}_{\Theta}+S\left(\hat{\omega}_{\Theta}\right) \mathbf{J}_{\Theta} \hat{\omega}_{\Theta}-f_{\omega_{\Theta}}\left(\hat{v}_{\Theta}, \hat{\omega}_{\Theta}, p, R\right)\right. \\
& +\left[0_{3 \times 1} \mathbf{J}_{\Theta}\right] \hat{h}_{6 \Theta}(\cdot)-\left[-S\left(M_{\Theta} \delta_{\Theta}\right)+\Gamma_{\Theta}(\cdot)^{\prime}\right] \mathbf{M}_{\Theta} \varphi_{\Theta} \\
& \left.-K_{z_{z_{\Theta}}} z_{2_{\Theta}}\right),
\end{aligned}
$$

the time derivative of $V_{3}$ becomes

$$
\begin{aligned}
\dot{V}_{3}= & -k_{e_{\Theta}} e^{\prime} \mathbf{M}_{\Theta}^{-1} e+e^{\prime} \delta_{\Theta}-e^{\prime} \tilde{v}_{\Theta}-\varphi_{\Theta}^{\prime} K_{\varphi_{\Theta}} \varphi_{\Theta} \\
& +\varphi_{\Theta}^{\prime} h_{3_{\Theta}}(\cdot)-z_{2_{\Theta}}^{\prime} K_{z_{2_{\Theta}}} z_{2_{\Theta}}+z_{2_{\Theta}}^{\prime} h_{4_{\Theta}}(\cdot) \\
& -\left(e^{\prime} R^{\prime} p_{d}^{\gamma}-\varphi_{\Theta}^{\prime} \mathbf{M}_{\Theta} h_{5_{\Theta}}(\cdot)+z_{2_{\Theta}}^{\prime}\left[0_{3 \times 1} \mathbf{J}_{\Theta}\right] \hat{h}_{7 \Theta}(\cdot)\right) z_{\gamma}
\end{aligned}
$$

where $\tilde{h}_{i_{\Theta}}:=\hat{h}_{i_{\Theta}}-h_{i_{\Theta}}, i=6,7, \hat{h}_{i_{\Theta}}$ is defined to be the same as $h_{i_{\Theta}}$, but substituting $v, \omega$ by $\hat{v}_{\Theta}, \hat{\omega}_{\Theta}$, and

$$
\begin{aligned}
h_{4_{\Theta}}\left(\hat{v}_{\Theta}, \hat{\omega}_{\Theta},\right. & v, \omega, p, R):=S\left(\hat{v}_{\Theta}\right) \mathbf{M}_{\Theta} \hat{v}_{\Theta}-S(v) \mathbf{M}_{\Theta} v \\
& +S\left(\hat{\omega}_{\Theta}\right) \mathbf{J}_{\Theta} \hat{\omega}_{\Theta}-S(\omega) \mathbf{J}_{\Theta} \omega+f_{\omega_{\Theta}}(v, \omega, p, R) \\
& -f_{\omega_{\Theta}}\left(\hat{v}_{\Theta}, \hat{\omega}_{\Theta}, p, R\right)-\mathbf{J}_{\Theta} L_{\omega} \tilde{\omega}_{\Theta}+\beta_{\omega_{\Theta}}(\cdot) \mathbf{J}_{\Theta} \tilde{\omega}_{\Theta} \\
& +\left[0_{3 \times 1} \mathbf{J}_{\Theta}\right]\left(\tilde{h}_{6 \Theta}(\cdot)+\tilde{h}_{7 \Theta}(\cdot) z_{\gamma}\right)
\end{aligned}
$$

Introduce now a forth control Lyapunov function given by

$V_{4}:=V_{3}+\frac{1}{2} z_{\gamma}^{2}=\frac{1}{2} e^{\prime} e+\frac{1}{2} \varphi_{\Theta}^{\prime} \mathbf{M}_{\Theta}^{2} \varphi_{\Theta}+\frac{1}{2} z_{2}^{\prime} \mathbf{J}_{\Theta} z_{2 \Theta}+\frac{1}{2} z_{\gamma}^{2}$.

Computing its time derivative, we get

$$
\begin{aligned}
\dot{V}_{4}= & -k_{e_{\Theta}} e^{\prime} \mathbf{M}_{\Theta}^{-1} e+e^{\prime} \delta_{\Theta}-e^{\prime} \tilde{v}_{\Theta}-\varphi_{\Theta}^{\prime} K_{\varphi_{\Theta}} \varphi_{\Theta}+\varphi_{\Theta}^{\prime} h_{3 \Theta}(\cdot) \\
& -z_{2_{\Theta}}^{\prime} K_{z_{2_{\Theta}}} z_{2_{\Theta}}+z_{2_{\Theta}}^{\prime} h_{4_{\Theta}}(\cdot)+z_{\gamma}\left(-e^{\prime} R^{\prime} p_{d}^{\gamma}\right. \\
& \left.+\varphi_{\Theta}^{\prime} \mathbf{M}_{\Theta} h_{5 \Theta}(\cdot)-z_{2_{\Theta}}^{\prime}\left[0_{3 \times 1} \mathbf{J}_{\Theta}\right] \hat{h}_{7 \Theta}(\cdot)+\ddot{\gamma}-v_{r}^{\gamma} \dot{\gamma}\right) .
\end{aligned}
$$

Selecting the following update law for $\ddot{\gamma}$ :

$$
\begin{aligned}
\ddot{\gamma}= & e^{\prime} R^{\prime} p_{d}^{\gamma}-\varphi_{\Theta}^{\prime} \mathbf{M}_{\Theta} h_{5_{\Theta}}(\cdot)+z_{2_{\Theta}}^{\prime}\left[0_{3 \times 1} \mathbf{J}_{\Theta}\right] \hat{h}_{7_{\Theta}}(\cdot) \\
& +v_{r}^{\gamma} \dot{\gamma}-k_{\gamma} z_{\gamma}
\end{aligned}
$$

where $k_{\gamma}$ is a positive constant, we obtain

$$
\dot{V}_{4}=-k_{e_{\Theta}} e^{\prime} \mathbf{M}_{\Theta}^{-1} e+e^{\prime} \delta_{\Theta}-e^{\prime} \tilde{v}_{\Theta}-\varphi_{\Theta}^{\prime} K_{\varphi_{\Theta}} \varphi_{\Theta}
$$




$$
+\varphi_{\Theta}^{\prime} h_{3_{\Theta}}(\cdot)-z_{2_{\Theta}}^{\prime} K_{z_{2_{\Theta}}} z_{2_{\Theta}}+z_{2_{\Theta}}^{\prime} h_{4_{\Theta}}(\cdot)-k_{\gamma} z_{\gamma}^{2} .
$$

An extension of Theorem 2 to the path-following then follows.

Theorem 3: Given a sufficiently smooth (with respect to $\gamma$ ) desired path $p_{d}(\gamma): \mathbb{R} \rightarrow \mathbb{R}^{3}$ with its derivatives (with respect to $\gamma$ ) bounded, and a desired assignment speed $v_{r}(\gamma)$ : $\mathbb{R} \rightarrow \mathbb{R}$, consider the hybrid system $\Sigma_{\text {path }}$ described by the underactuated vehicle model (2)-(3) in closed-loop with the switched multi-controller (22), (38), (39), the multi-estimator (16), and the switching logic described in Section IV-C.

i) For any initial condition of $\Sigma_{\text {path }}$ with $\mu_{\Theta}(0)>0, \forall \Theta \in$ $\mathcal{P}$, the solution exists globally and all closed-loop signals are bounded.

ii) There exist a finite time $T^{\dagger} \geq 0$ such that $\sigma(t)=$ $\Theta^{\dagger} \in \mathcal{P}$ for all $t \geq T^{\dagger}$ (i.e., the switching stops in finite time). Moreover, as $t \rightarrow \infty$, the position error $\left\|p(t)-p_{d}(\gamma(t))\right\|$ and the speed error $\left\|\dot{\gamma}(t)-v_{r}(\gamma(t))\right\|$ converge to neighborhoods of the origin that can be made arbitrarily small by appropriate choice of the control parameters.

Proof: The proof is not given since it is a simple application of the arguments used in the previous theorems.

\section{APPLICATION TO SPECIFIC VEHICLES}

This section illustrates the application of the previous results to two vehicles: a hovercraft (moving on a planar surface) and an underwater vehicle (moving in three-dimensional space).

\section{A. Trajectory-tracking of an underactuated Hovercraft}

Consider the Caltech MVWT vehicle described in [43], [47] consisting of a platform mounted on three low-friction, omnidirectional casters, with two attached high-performance ducted fans. Let $p=(x, y)^{\prime} \in \mathbb{R}^{2}$ be the Cartesian coordinates of the vehicle's center of mass and $\theta \in \mathbb{S}^{1}$ its orientation. Assuming that the friction and moment forces can be modeled by viscous friction, the equations of motion are

$$
\begin{aligned}
m \ddot{x} & =-d_{v} \dot{x}+\left(F_{s}+F_{p}\right) \cos \theta \\
m \ddot{y} & =-d_{v} \dot{y}+\left(F_{s}+F_{p}\right) \sin \theta \\
J \ddot{\theta} & =-d_{\omega} \dot{\theta}+l\left(F_{s}-F_{p}\right)
\end{aligned}
$$

where $m=5.5 \mathrm{~kg}$ is the mass of the vehicle and $J=$ $0.047 \mathrm{Kg} \mathrm{m}^{2}$ is the rotational inertia. The starboard and portboard fan forces are denoted $F_{s}$ and $F_{p}$, respectively, and $l=0.123 \mathrm{~m}$ denotes the moment arm of the forces. The geometric and mass centers of the vehicle are assumed to coincide. The coefficient of viscous friction $d_{v}$ is $5.5 \mathrm{Kg} / \mathrm{s}$ and the coefficient of rotational friction $d_{\omega}$ is $0.41 \mathrm{Kg} \mathrm{m} / \mathrm{s}$. Expressing the equations of motion in the body fixed frame, yields (2)-(3) with $(R, p) \in S E(2), v \in \mathbb{R}^{2}, \omega \in \mathbb{R}, R(\theta)=$ $\left(\begin{array}{cc}\cos \theta & -\sin \theta \\ \sin \theta & \cos \theta\end{array}\right), S(\omega)=\left(\begin{array}{cc}0 & -\omega \\ \omega & 0\end{array}\right), \mathbf{M}=\operatorname{diag}\{m, m\}, \mathbf{J}=J$, $f_{v}=-\operatorname{diag}\left\{d_{v}, d_{v}\right\} v, f_{\omega}=-d_{\omega} \omega, g_{v}=(1,0)^{\prime}, G_{\omega}=1$, $u_{v}=F_{s}+F_{p}$, and $u_{\omega}=l\left(F_{s}-F_{p}\right)$. In this case the matrix B introduced in (8) is given by $\mathrm{B}=\left(\begin{array}{cc}1 & m \delta_{2} \\ 0 & -m \delta_{1}\end{array}\right)$ with $\delta=\left(\delta_{1}, \delta_{2}\right)^{\prime}$. The reader is referred to [43] for a detailed coverage of the trajectory-tracking controller with experimental results.

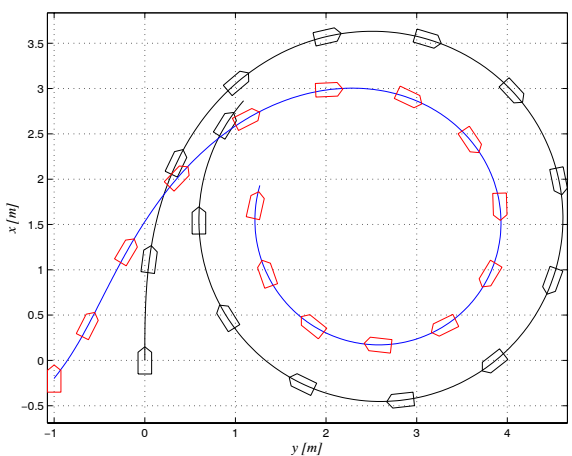

(a)

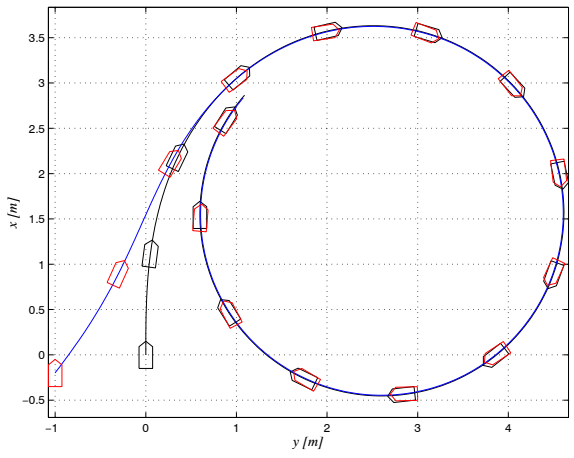

(b)
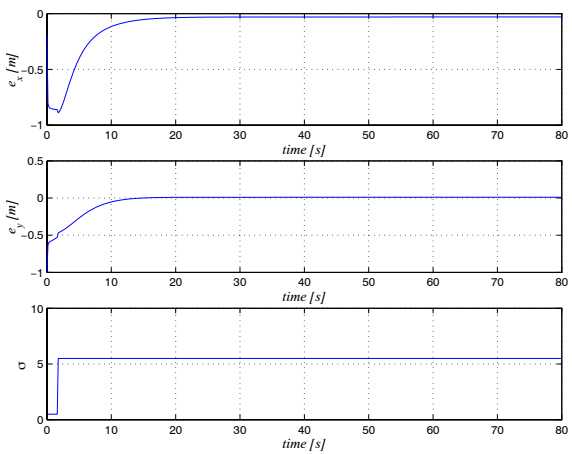

(c)

Fig. 2. First experiment. Trajectory of the hovercraft in the $x y$-plane and reference trajectory performed by a unicycle vehicle using the trajectorytracking controller presented in Section III (diagram (a)) and the estimatorbased supervisory controller for trajectory-tracking (diagram (b)). Time evolution of the tracking error in $x$-direction, in $y$-direction, and the switching signal $\sigma$ for the estimator-based supervisory controller (diagram (c)).

We now describe two simulation results that illustrate the performance of the proposed tracking controller with and without supervisory control. The objective of the first experiment is to force the hovercraft to track the "virtual" kinematic unicycle vehicle

$$
\dot{x}_{d}=V_{d} \cos \theta_{d}, \quad \dot{y}_{d}=V_{d} \sin \theta_{d}, \quad \dot{\theta}_{d}=\omega_{d},
$$

which starts at $x_{d}(0)=y_{d}(0)=\theta_{d}(0)=0$ and moves with velocities $V_{d}(t)=0.2 \mathrm{~m} / \mathrm{s}$ and $\omega_{d}(t)=0.1 \mathrm{rad} / \mathrm{s}$. The initial conditions for the hovercraft are $\left(x_{0}, y_{0}\right)=(-0.2 m,-1 m)$, $R_{0}=I, v_{0}=\omega_{0}=0$. For simplicity, only the coefficient of viscous friction is unknown, but assumed to belong to the set $\mathcal{P}=\{0.5,1.0, \ldots, 9.5,10.0\}$. The control parameters were 


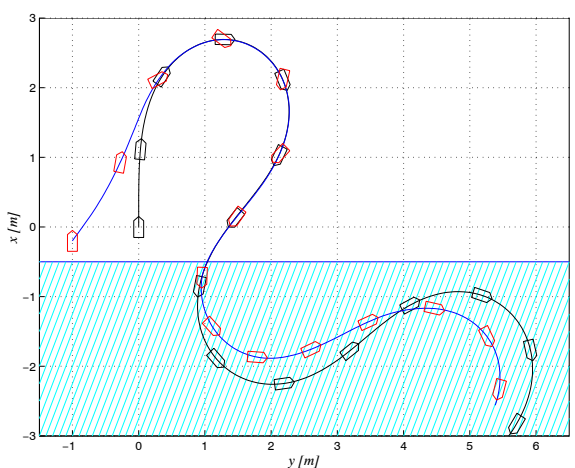

(a)

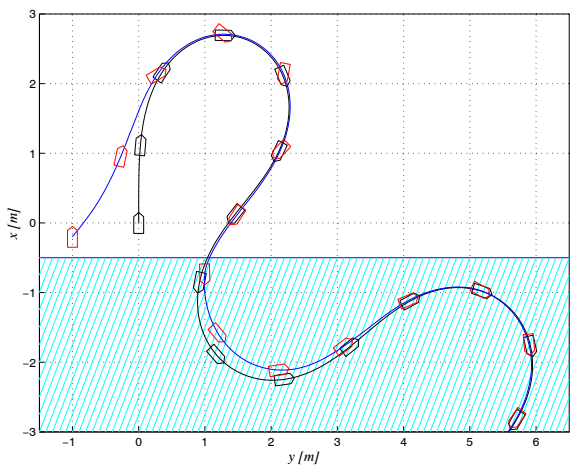

(b)
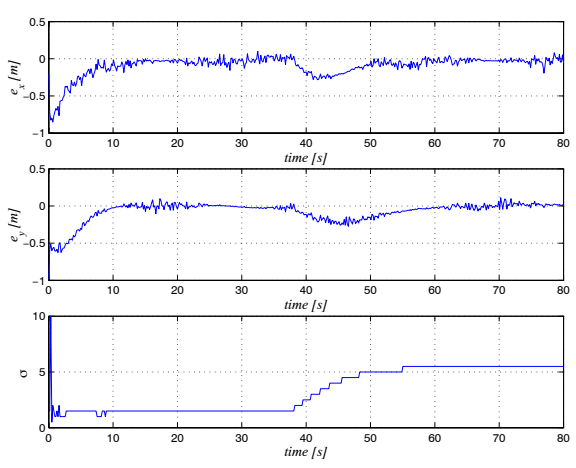

(c)

Fig. 3. Second experiment. Trajectory of the hovercraft in the $x y$-plane and reference trajectory performed by a unicycle vehicle using the trajectorytracking controller presented in Section III (diagram (a)) and the estimatorbased supervisory controller for trajectory-tracking (diagram (b)). Time evolution of the tracking error in $x$-direction, in $y$-direction, and the switching signal $\sigma$ for the estimator-based supervisory controller (diagram (c)).

selected as follows: $k_{e_{\Theta}}=1.5, K_{\varphi_{\Theta}}=8 I, K_{z_{2_{\Theta}}}=8$, and $\delta_{\Theta}=(-0.01,0)^{\prime}$ for all $\Theta \in \mathcal{P}$. The hysteresis constant for the switching logic was set to $h=0.1$, the forgetting factor to $\lambda_{\mu}=1.0$, and the multi-estimator gains to $L_{v}=0.1 I$ and $L_{\omega}=0.1$. The functions $\beta_{v}(\cdot)$ and $\beta_{\omega}(\cdot)$ introduced in (16) are given by $\beta_{v}(\cdot)=\beta_{\omega}(\cdot)=\omega^{2}$.

To illustrate the benefits derived from the supervisory control scheme proposed in Section IV, we show in Fig. 2(a) the closed-loop trajectory for the (non-adaptive) trajectorytracking controller presented in Section III when the value of the coefficient of viscous friction assumed by the control system was set to $10 \%$ of the real value. It can be seen that although the closed-loop is still stable, the parameter error affects considerably the closed-loop performance. In contrast, Fig. 2(b) shows the closed-loop trajectory for the supervisory controller where, as expected, the hovercraft converges to a small neighborhood of the "virtual" unicycle vehicle, in spite of the uncertainty in $d_{v}$. Fig. 2(c) shows the time evolution of some relevant variables. In steady-state the vehicle is not aligned with the direction of the tangent velocity of $p_{d}$. Contrary to what happens for wheeled mobile robots (with inherent lateral drag coefficient $d_{v}=+\infty$ ) in the hovercraft case we cannot force the orientation $\theta$ to converge to the direction of the tangent velocity $p_{d}$.

To further illustrate the usefulness of the adaptive scheme and test its robustness with respect to sensor noise, a second experiment is described. In this case, all the initial conditions and control parameters are as in the first experiment, but now the "virtual" unicycle vehicle moves with linear velocity $V_{d}=$ $0.2 \mathrm{~m} / \mathrm{s}$ and angular velocity $\omega_{d}$ such that

$$
\dot{\omega}_{d}=-0.1\left(\omega_{d}+0.3 \sin (t / 8)\right), \quad \omega_{d}(0)=0 .
$$

Zero mean uniform random noise was introduced in every sensed signal: the measured velocities $v$, and $\omega$; the orientation angle $\theta$; and the $x$ and $y$ positions. The amplitude was set to $(0.05,0.05), 0.05,0.1,0.1$, and 0.1 , respectively. We also consider the situation where the Hovercraft moves between two surfaces characterized by distinct friction coefficients (e.g., from water to land). To simulate this effect, we set the value of the coefficient of viscous friction to $d_{v}=1.5 \mathrm{Kg} / \mathrm{s}$ while the Hovercraft is in the region $\left\{(x, y) \in \mathbb{R}^{2}: x \geq-0.5\right\}$ and $d_{v}=5.5 \mathrm{Kg} / \mathrm{s}$, otherwise. We can see in Fig. 3(b) that the hovercraft still converges to a very small neighborhood of the target unicycle vehicle and its performance is not significantly affected by the switching in $d_{v}$.

\section{B. Trajectory-tracking and path-following of an underwater vehicle in 3-D space}

Consider an ellipsoidal shaped underactuated autonomous underwater vehicle (AUV) not necessarily neutrally buoyant. Let $\{\mathcal{B}\}$ be a body-fixed coordinate frame whose origin is located at the center of mass of the vehicle and suppose that we have available a pure body-fixed control force $\tau_{u}$ in the $x_{B}$ direction, and two independent control torques $\tau_{q}$ and $\tau_{r}$ about the $y_{B}$ and $z_{B}$ axes of the vehicle, respectively. The kinematics and dynamics equations of motion of the vehicle can be written as (2)-(3), where $\mathbf{M}=\operatorname{diag}\left\{m_{11}, m_{22}, m_{33}\right\}$, $\mathbf{J}=\operatorname{diag}\left\{J_{11}, J_{22}, J_{33}\right\}, u_{v}=\tau_{u}, u_{\omega}=\left(\tau_{q}, \tau_{r}\right)^{\prime}, D_{v}(v)=$ $\operatorname{diag}\left\{X_{v_{1}}+X_{\left|v_{1}\right| v_{1}}\left|v_{1}\right|, Y_{v_{2}}+Y_{\left|v_{2}\right| v_{2}}\left|v_{2}\right|, Z_{v_{3}}+Z_{\left|v_{3}\right| v_{3} \mid}\left|v_{3}\right|\right\}$, $D_{\omega}(\omega)=\operatorname{diag}\left\{K_{\omega_{1}}+K_{\left|\omega_{1}\right| \omega_{1}}\left|\omega_{1}\right|, M_{\omega_{2}}+M_{\left|\omega_{2}\right| \omega_{2}}\left|\omega_{2}\right|, N_{\omega_{3}}+\right.$

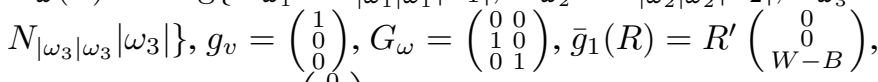
$\bar{g}_{2}(R)=S\left(r_{B}\right) R^{\prime}\left(\begin{array}{l}0 \\ 0 \\ B\end{array}\right) f_{v}=-D_{v}(v) v-\bar{g}_{1}(R), f_{\omega}=$ $-D_{\omega}(\omega) \omega-\bar{g}_{2}(R)$. The gravitational and buoyant forces are given by $W=m g$ and $B=\rho g \nabla$, respectively, where $m$ is the mass, $\rho$ is the mass density of the water and $\nabla$ is the volume of the displaced water. The numerical values used for the physical parameters match those of the Sirene AUV, described in [48], [49]. The matrix B defined in (8) 
takes the form $\mathrm{B}=\left[b_{1}, b_{2}, b_{3}, b_{4}\right]$, where $b_{1}:=[1,0,0]^{\prime}$, $b_{2}:=\left[0, m_{33} \delta_{3}+a_{3}\left(m_{33}-m_{22}\right),-m_{22} \delta_{2}-a_{2}\left(m_{22}-m_{33}\right)\right]^{\prime}$, $b_{3}:=\left[-m_{33} \delta_{3}-a_{3}\left(m_{33}-m_{11}\right), 0, m_{11} \delta_{1}+a_{1}\left(m_{11}-m_{33}\right)\right]^{\prime}$, $b_{4}:=\left[m_{22} \delta_{2}+a_{2}\left(m_{22}-m_{11}\right),-m_{11} \delta_{1}+a_{1}\left(m_{11}-m_{22}\right), 0\right]^{\prime}$, $a=\left(a_{1}, a_{2}, a_{3}\right)^{\prime}:=R^{\prime} \dot{p}_{d}$.

Two simulation results are included to illustrate the dynamic behavior of the AUV in closed-loop with the trajectorytracking controller presented in Section III, and the pathfollowing controller in Section V. Fig. 4(a) displays the vehicle trajectory using the trajectory-tracking controller in the 3Dspace for the following desired trajectory

$$
p_{d}(t)=\left[V_{1} \cos \left(\frac{2 \pi}{T} t+\phi_{d}\right), V_{1} \sin \left(\frac{2 \pi}{T} t+\phi_{d}\right), V_{2} t\right]
$$

with $V_{1}=20, V_{2}=0.05, T=400$, and $\phi_{d}=-\frac{\pi}{2}$. The initial conditions of the AUV are $p_{0}=\left(x_{0}, y_{0}, z_{0}\right)=$ $(10 \mathrm{~m},-10 \mathrm{~m}, 0), R=I$, and $v_{0}=\omega_{0}=0$. The control parameters were selected as follows: $k_{e}=0.1, K_{\varphi}=I$, $K_{z_{2}}=I$, and $\delta=\left(-2 \max \left(\left|m_{11}-m_{22}\right|,\left|m_{11}-m_{33}\right|\right), 0,0\right)^{\prime}$. Figures 4(c) and 4(e) show the time evolution of the tracking error $e$ and the Euler angles (computed from $R$ ), respectively. The damped oscillatory behavior of pitch and roll are due to the gravitational and buoyancy forces. Notice that the initial position of the desired position was deliberately chosen to be almost behind the initial position of the vehicle. As we can see, the vehicle turns back in its attempt to be at the given reference position at the prescribed time, requiring significant control effort and consequently inducing a strong oscillatory behavior. The path-following controller in Section $\mathrm{V}$ was used to generate the trajectories in Fig. 4(b), 4(d), and 4(f) where the desired path, the initial conditions and the control gains are the same as in the experiment for the tracking controller. The guidance gain and the speed assignment were set to $k_{\gamma}=2$ and $v_{r}=1 \mathrm{~m} / \mathrm{s}$, respectively. The initial condition for $\gamma$ was chosen to be the one that minimizes the distance between the initial position of the AUV and the desired path. The convergence of the vehicle to the path is now much smoother. From these two experiments one can see that when the primary objective is to steer the vehicle to converge to and move along a geometric path, the path-following controller offers significant performance improvement. For simplicity, in these experiments we did not include model uncertainty.

\section{CONCLUSIONS}

We proposed a solution to the trajectory-tracking and pathfollowing problem for underactuated autonomous vehicles in the presence of possibly large modeling parametric uncertainty. For a general class of vehicles moving in either two or three-dimensional space, we demonstrated how adaptive switching supervisory control can be combined with a nonlinear Lyapunov-based tracking control law to design a hybrid controller that yields global boundedness and convergence of the position tracking error to a small neighborhood, and robustness to parametric modeling uncertainty. We illustrated our results in the context of two vehicle control applications: a hovercraft (moving on a planar surface) and an underwater vehicle (moving in three-dimensional space). Simulations show that the control objectives were accomplished.
An alternative approach to the Lyapunov-based control scheme proposed in Section III consists in choosing an adequate point linked to the vehicle and then utilize output feedback linearization to design a simple controller that drives that point to the reference trajectory. See for example [10] for the case of unicycle-like mobile robots. For general underactuated vehicles, the stability of the zero-dynamics would have to be established independently. This is an issue for future research.

A problem that warrants further research is the control of underactuated vehicles with noise and in the presence of disturbances. Typical disturbances for marine vehicles include the ones induced by wave, wind, and ocean current.

\section{APPENDIX}

Property 1: Let $g_{v}=\left(g_{v_{1}}, g_{v_{2}}, g_{v_{3}}\right)^{\prime}$ be non-zero and $\dot{p}_{d}$ uniformly bounded by $\left\|\dot{p}_{d}\right\| \leq \gamma_{\dot{p}_{d}}$. Then, there exists a vector $\delta$ that makes $\mathrm{B}\left(R, \dot{p}_{d}, \delta\right)$ defined in (8) full-rank.

Proof: Pick $\delta=\mathbf{M}^{-1} \mu \varepsilon$, where $\mu=\left(\mu_{1}, \mu_{2}, \mu_{3}\right)^{\prime}:=$ $(1,0,0)^{\prime}$ and $\varepsilon$ is a positive constant to be selected shortly. Defining $\Delta:=\frac{1}{\varepsilon} \Gamma$, we conclude that

$$
\begin{aligned}
& \operatorname{rank} \mathrm{B}=\operatorname{rank}\left[g_{v} \varepsilon S(\mu)+\Gamma\right]\left[\begin{array}{cc}
I & 0 \\
0 & \frac{1}{\varepsilon} I
\end{array}\right] \\
& =\operatorname{rank}\left[g_{v} S(\mu)+\Delta\right] \\
& =\operatorname{rank}\left[\begin{array}{cccc}
g_{v_{1}} & \Delta_{11} & -\mu_{3}+\Delta_{12} & \mu_{2}+\Delta_{13} \\
g_{v_{2}} & \mu_{3}+\Delta_{21} & \Delta_{22} & -\mu_{1}+\Delta_{23} \\
g_{v_{3}} & -\mu_{2}+\Delta_{31} & \mu_{1}+\Delta_{32} & \Delta_{33}
\end{array}\right]
\end{aligned}
$$

where we used the fact that the rank of a matrix does not change when it is multiplied by a non-singular matrix. From the definition of $\Gamma$ (see (6)) we conclude that each element of $\Delta$ can be bounded by $\left|\Delta_{i j}\right| \leq \frac{2 \lambda_{\max }(\mathbf{M}) \gamma_{\dot{p}_{d}}}{\varepsilon}$. Much tighter bounds can be obtained for specific class of vehicles. For example when $\mathbf{M}=m I$ then $\Gamma=\Delta=0$. Assume now that $g_{v_{1}} \neq 0$. From (40), we conclude that B has full-rank if one can find at least one $3 \times 3$ minor determinant of $\left[\begin{array}{ll}g_{v} & S(\mu)+\Delta\end{array}\right]$ non-zero. Consider the one formed by the first, third and forth columns, i.e.,

$$
\begin{aligned}
\left|\begin{array}{ccc}
g_{v_{1}} & \Delta_{12} & \Delta_{13} \\
g_{v_{2}} & \Delta_{22} & -\mu_{1}+\Delta_{23} \\
g_{v_{3}} & \mu_{1}+\Delta_{32} & \Delta_{33}
\end{array}\right|= & \mu_{1}\left[\mu_{1} g_{v_{1}}+g_{v_{1}} \Delta_{32}-g_{v_{1}} \Delta_{23}\right. \\
& \left.+g_{v_{2}} \Delta_{13}-g_{v_{3}} \Delta_{12}\right]+h_{\Delta}
\end{aligned}
$$

where $h_{\Delta}$ collects all the remaining terms and does not depend on $\mu_{1}$. It follows now from the fact that each $\Delta_{i j}$ can be arbitrarily small by choosing $\varepsilon$ (and consequently $\delta$ ) sufficient large, that (41) can be made nonzero and therefore B full-rank. If $g_{v_{1}}=0$, the same conclusion about the full-rank of B can be made by following the same reasoning but with $\mu=(0,1,0)^{\prime}$ if $g_{v_{2}} \neq 0$, or $\mu=(0,0,1)^{\prime}$ for the case $g_{v_{1}}=g_{v_{2}}=0$.

\section{Lemma 1}

Proof: Throughout this proof, to avoid cumbersome notation, we will use $\hat{v}, \tilde{v}, \hat{\omega}, \tilde{\omega}$ to denote $\hat{v}_{\Theta^{*}}, \tilde{v}_{\Theta^{*}}, \hat{\omega}_{\Theta^{*}}$, and $\tilde{\omega}_{\Theta^{\star}}$, respectively. Note also that $\Theta=\Theta^{\star}$ corresponds to the nominal model and therefore $\mathbf{M}_{\Theta^{\star}}$ is equal to $\mathbf{M}$. The same applies for the other model parameters. Consider the following exponentially weighted Lyapunov-like functions

$$
V_{v}:=\frac{1}{2} e^{2 \lambda_{\mu} t}\|\tilde{v}\|^{2}, \quad V_{\omega}:=\frac{1}{2} e^{2 \lambda_{\mu} t}\|\tilde{\omega}\|^{2}
$$




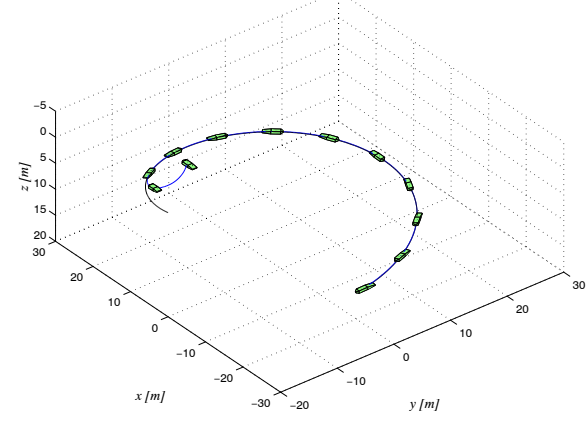

(a)
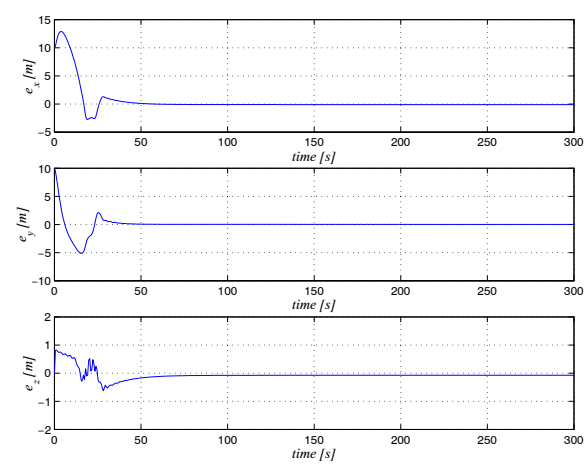

(c)
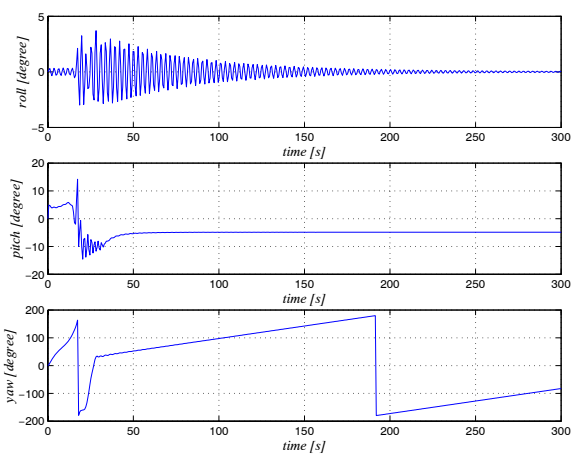

(e)

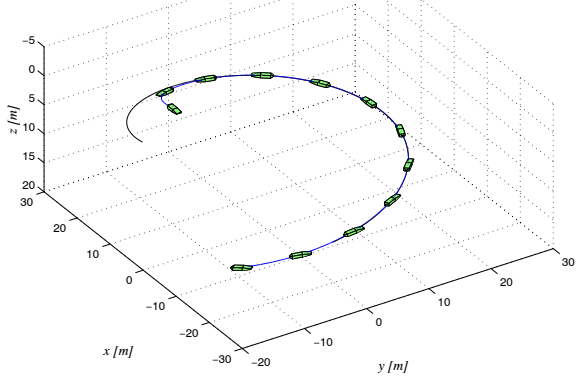

(b)

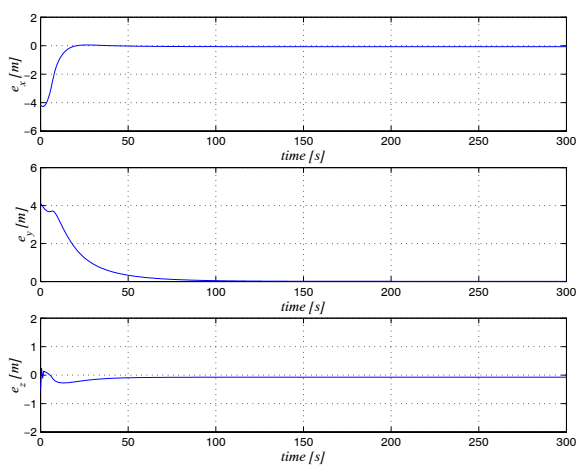

(d)
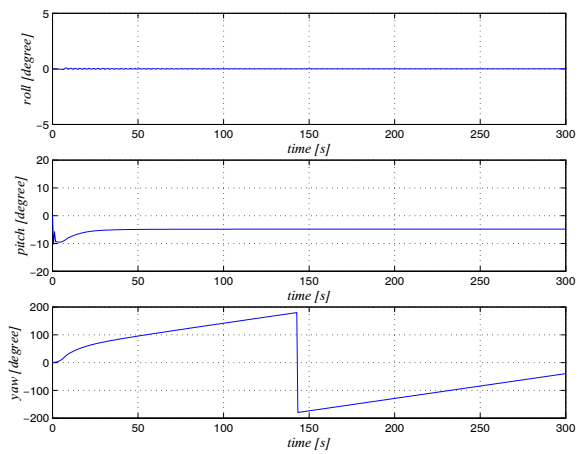

(f)

Fig. 4. Vehicle trajectory in 3-D space using the trajectory-tracking controller presented in Section III (diagram (a)), and the path-following controller (diagram (b)). Time evolution of the position error $e=\left(e_{x}, e_{y}, e_{z}\right)$, the roll $\phi$, pitch $\theta$, and yaw $\psi$ Euler angles for the trajectory-tracking (diagrams (c), (e)), and the path-following (diagrams (d), (f)).

where $\lambda_{\mu}$ is any positive constant that satisfies

$$
\lambda_{\mu}<\min \left\{\lambda_{\min }\left(L_{v}\right), \lambda_{\min }\left(L_{\omega}\right)\right\} .
$$

Computing the time derivative of $V_{v}$ and $V_{\omega}$ along the solutions of (2b), (3), and (16) for $\Theta=\Theta^{\star}$, we obtain

$$
\begin{aligned}
\dot{V}_{v}= & -e^{2 \lambda_{\mu} t}\left[\tilde{v}^{\prime} L_{v} \tilde{v}-\lambda_{\mu}\|\tilde{v}\|^{2}\right] \\
& -e^{2 \lambda_{\mu} t} \beta_{v_{\Theta^{\star}}}(\hat{v}, \hat{\omega}, v, \omega, p, R)\|\tilde{v}\|^{2} \\
\dot{V}_{\omega}= & -e^{2 \lambda_{\mu} t}\left[\tilde{\omega}^{\prime} L_{\omega} \tilde{\omega}-\lambda_{\mu}\|\tilde{\omega}\|^{2}\right] \\
& -e^{2 \lambda_{\mu} t} \beta_{\omega_{\Theta^{*}}}(\hat{v}, \hat{\omega}, \omega, p, R)\|\tilde{\omega}\|^{2} .
\end{aligned}
$$

To prove (18a), consider the Lyapunov function $V:=$ $\frac{1}{2}\left\|\mathbf{e}_{\Theta^{*}}\right\|^{2}$, which is the same as $V=V_{v}+V_{\omega}$ with $\lambda_{\mu}=0$. From (43) it follows that $\dot{V} \leq$ $-2 \min \left\{\lambda_{\min }\left(L_{v}\right), \lambda_{\min }\left(L_{\omega}\right)\right\} V$. Using the Comparison
Lemma [45] one concludes that (18a) holds with $\kappa=$ $\min \left\{\lambda_{\min }\left(L_{v}\right), \lambda_{\min }\left(L_{\omega}\right)\right\}$ and $\gamma_{1}=\left\|\mathbf{e}_{\Theta^{*}}(0)\right\|$.

To prove (18b), observe from (43a) and (42) that

$$
\begin{aligned}
\dot{V}_{v} & \leq-e^{2 \lambda_{\mu} t} \beta_{v_{\Theta^{\star}}}(\hat{v}, \hat{\omega}, v, \omega, p, R)\|\tilde{v}\|^{2} \\
& \leq-2 \beta_{v_{\Theta^{\star}}}(\hat{v}, \hat{\omega}, v, \omega, p, R) V_{v} .
\end{aligned}
$$

Defining $W_{v}:=\sqrt{V_{v}}=\frac{1}{\sqrt{2}} e^{\lambda_{\mu} t}\|\tilde{v}\|$ and taking an interval $[0, \tau)$ on which $V_{v}>0$, we have $\dot{W}_{v} \leq$ $-\beta_{v_{\Theta^{\star}}}(\hat{v}, \hat{\omega}, v, \omega, p, R) W_{v}, t \in[0, \tau)$ and therefore

$$
\begin{array}{r}
W_{v}(t)-W_{v}(0) \leq-\int_{0}^{t} \beta_{v_{\Theta^{*}}}(\hat{v}(\tau), \hat{\omega}(\tau), v(\tau), \omega(\tau), p(\tau), \\
R(\tau)) W_{v}(\tau) d \tau, \quad t \in[0, \tau)
\end{array}
$$


Consequently,

$$
\begin{aligned}
& \int_{0}^{t} e^{\lambda_{\mu} \tau} \beta_{v_{\Theta^{*}}}(\hat{v}(\tau), \hat{\omega}(\tau), v(\tau), \omega(\tau), \\
&p(\tau), R(\tau))\|\tilde{v}(\tau)\| d \tau \leq \gamma_{2}<\infty, \quad t \in[0, \tau)
\end{aligned}
$$

with $\gamma_{2}=\|\tilde{v}(0)\|$. On the other hand, if $V_{v}(\tau)=0$ for some $\tau>0, V_{v}(t)=0$ for all $t \geq \tau$. Therefore,

$$
\begin{aligned}
& \int_{0}^{t} e^{\lambda_{\mu} \tau} \beta_{v_{\Theta^{\star}}}(\hat{v}(\tau), \hat{\omega}(\tau) v(\tau), \omega(\tau), p(\tau), R(\tau))\|\tilde{v}(\tau)\| d \tau \\
&=\int_{0}^{\tau} e^{\lambda_{\mu} \tau} \beta_{v_{\Theta^{\star}}}(\hat{v}(\tau), \hat{\omega}(\tau), v(\tau), \omega(\tau), \\
&p(\tau), R(\tau))\|\tilde{v}(\tau)\| d \tau \leq \gamma_{2}<\infty
\end{aligned}
$$

Inequality (18c) can be also concluded by applying the same arguments to (43b). In that case $\gamma_{3}=\|\tilde{\omega}(0)\|$.

\section{Lemma 2}

Proof: Taking norms to $h_{3 \Theta}(\cdot)$ defined in (20) and using (17a) we conclude that

$$
\begin{aligned}
\left\|h_{3_{\Theta}}(\cdot)\right\| \leq & {\left[\left\|\mathbf{M}_{\Theta}^{2} L_{v}\right\|_{2}+k_{e_{\Theta}} \lambda_{\max }\left(\mathbf{M}_{\Theta}\right)\right]\left\|\tilde{v}_{\Theta}\right\| } \\
& +\lambda_{\max }\left(\mathbf{M}_{\Theta}\right) \beta_{v_{\Theta}}(\cdot)\left[\lambda_{\max }\left(\mathbf{M}_{\Theta}\right)+c_{1}\right]\left\|\tilde{v}_{\Theta}\right\| \\
& +\lambda_{\max }\left(\mathbf{M}_{\Theta}\right)\left[\left\|\mathbf{M}_{\Theta} \delta_{\Theta}\right\|\right. \\
& \left.+2 \lambda_{\max }\left(\mathbf{M}_{\Theta}\right)\left\|\dot{p}_{d}\right\|\right]\left\|\tilde{\omega}_{\Theta}\right\|
\end{aligned}
$$

Also, from (17b)-(17c), a bound for $h_{4_{\Theta}}(\cdot)$ given in (27) can be computed as follows.

$$
\begin{aligned}
\left\|h_{4_{\Theta}}(\cdot)\right\| \leq & \| S\left(\hat{v}_{\Theta}\right) \mathbf{M}_{\Theta} \hat{v}_{\Theta}-S\left(\hat{v}_{\Theta}\right) \mathbf{M}_{\Theta} v+S\left(\hat{v}_{\Theta}\right) \mathbf{M}_{\Theta} v \\
& -S(v) \mathbf{M}_{\Theta} v\|+\| S\left(\hat{\omega}_{\Theta}\right) \mathbf{J}_{\Theta} \hat{\omega}_{\Theta}-S\left(\hat{\omega}_{\Theta}\right) \mathbf{J}_{\Theta} \omega \\
& +S\left(\hat{\omega}_{\Theta}\right) \mathbf{J}_{\Theta} \omega-S(\omega) \mathbf{J}_{\Theta} \omega\|+\| f_{\omega_{\Theta}}(v, \omega, p, R) \\
& -f_{\omega_{\Theta}}\left(\hat{v}_{\Theta}, \omega, p, R\right)+f_{\omega_{\Theta}}\left(\hat{v}_{\Theta}, \omega, p, R\right) \\
& -f_{\omega_{\Theta}}\left(\hat{v}_{\Theta}, \hat{\omega}_{\Theta}, p, R\right) \|+\left[\left\|\mathbf{J}_{\Theta} L_{\omega}\right\|_{2}\right. \\
& \left.+\beta_{\omega_{\Theta}}(\cdot) \lambda_{\max }\left(\mathbf{J}_{\Theta}\right)\right] \tilde{\omega}_{\Theta}+\left\|\left[0_{3 \times 1} \mathbf{J}_{\Theta}\right] \varphi_{v_{\Theta}}(\cdot)\right\| \\
& +\left\|\left[0_{3 \times 1} \mathbf{J}_{\Theta}\right] \varphi_{\omega_{\Theta}}(\cdot)\right\| \\
\leq & c_{2} \beta_{2_{\Theta}}\left(\hat{v}_{\Theta}, \hat{\omega}_{\Theta}, v, \omega, p, R\right)\left\|\tilde{v}_{\Theta}\right\|+\left[\left(\lambda_{\max }\left(\mathbf{J}_{\Theta}\right)\right.\right. \\
& \left.\left.+c_{3}\right) \beta_{\omega_{\Theta}}\left(\hat{v}_{\Theta}, \hat{\omega}_{\Theta}, \omega, p, R\right)+\left\|\mathbf{J}_{\Theta} L_{\omega}\right\|_{2}\right]\left\|\tilde{\omega}_{\Theta}\right\|
\end{aligned}
$$

Using these two bounds in (26) and resorting to Young's inequality it follows that for every positive constant $\gamma_{i}, i=$ $1, \ldots, 9, \dot{V}_{3}$ satisfies

$$
\begin{aligned}
\dot{V}_{3} \leq & -\left[k_{e_{\Theta}} \lambda_{\min }\left(\mathbf{M}_{\Theta}^{-1}\right)-\frac{\gamma_{1}}{2}-\frac{\gamma_{2}}{2}\right]\|e\|^{2} \\
& -\left[\lambda_{\min }\left(K_{\varphi_{\Theta}}\right)-\frac{\gamma_{3}}{2}\left\|\mathbf{M}_{\Theta}^{2} L_{v}\right\|_{2}+k_{e_{\Theta}} \lambda_{\max }\left(\mathbf{M}_{\Theta}\right)\right] \\
& -\frac{\gamma_{4}}{2} \lambda_{\max }\left(\mathbf{M}_{\Theta}\right) \beta_{v_{\Theta}}(\cdot)\left(\lambda_{\max }\left(\mathbf{M}_{\Theta}\right)+c_{1}\right)\left\|\tilde{v}_{\Theta}\right\| \\
& \left.-\frac{\gamma_{5}}{2} \lambda_{\max }\left(\mathbf{M}_{\Theta}\right)\left[\left\|\mathbf{M}_{\Theta} \delta_{\Theta}\right\|+2 \lambda_{\max }\left(\mathbf{M}_{\Theta}\right)\left\|\dot{p}_{d}\right\|\right]\right]\left\|\varphi_{\Theta}\right\|^{2} \\
& +\left[\frac{1}{2 \gamma_{2}}+\frac{1}{2 \gamma_{3}}\left[\left\|\mathbf{M}_{\Theta}^{2} L_{v}\right\|_{2}+k_{e_{\Theta}} \lambda_{\max }\left(\mathbf{M}_{\Theta}\right)\right]\right]\left\|\tilde{v}_{\Theta}\right\|^{2} \\
& +\left[\frac{1}{2 \gamma_{5}} \lambda_{\max }\left(\mathbf{M}_{\Theta}\right)\left(\left\|\mathbf{M}_{\Theta} \delta_{\Theta}\right\|+2 \lambda_{\max }\left(\mathbf{M}_{\Theta}\right)\left\|\dot{p}_{d}\right\|\right)\right. \\
& \left.+\frac{1}{2 \gamma_{9}}\left\|\mathbf{J}_{\Theta} L_{\omega}\right\|_{2}\right]\left\|\tilde{\omega}_{\Theta}\right\|^{2}+\left[\frac{1}{2 \gamma_{4}} \lambda_{\max }\left(\mathbf{M}_{\Theta}\right) \beta_{v_{\Theta}}(\cdot)\right.
\end{aligned}
$$

$$
\begin{aligned}
& \left.\times\left(\lambda_{\max }\left(\mathbf{M}_{\Theta}\right)+c_{1}\right)+\frac{1}{2 \gamma_{7}} c_{2} \beta_{2_{\Theta}}(\cdot)\right]\left\|\tilde{v}_{\Theta}\right\| \\
& +\frac{1}{2 \gamma_{8}}\left(\lambda_{\max }(\mathbf{J})+c_{3}\right) \beta_{\omega_{\Theta}}(\cdot)\left\|\tilde{\omega}_{\Theta}\right\| \\
& -\left[\lambda_{\min }\left(K_{z_{2_{\Theta}}}\right)-\frac{\gamma_{7}}{2} c_{2} \beta_{2_{\Theta}}(\cdot)\left\|\tilde{v}_{\Theta}\right\|-\frac{\gamma_{8}}{2}\left(\lambda_{\max }\left(\mathbf{J}_{\Theta}\right)\right.\right. \\
& \left.\left.+c_{3}\right) \beta_{\omega_{\Theta}}(\cdot)\left\|\tilde{\omega}_{\Theta}\right\|-\frac{\gamma_{9}}{2}\left\|\mathbf{J}_{\Theta} L_{\omega}\right\|_{2}\right]\left\|z_{2_{\Theta}}\right\|^{2}+\frac{1}{2 \gamma_{1}}\left\|\delta_{\Theta}\right\|^{2}
\end{aligned}
$$

where we used the facts that $\left\|\tilde{v}_{\Theta}\right\|\left\|\varphi_{\Theta}\right\| \leq\left\|\tilde{v}_{\Theta}\right\|\left[\frac{1}{2 \gamma_{4}}+\right.$ $\left.\frac{\gamma_{4}}{2}\|\varphi\|^{2}\right]$ and $\left\|\tilde{\omega}_{\Theta}\right\|\left\|z_{2_{\Theta}}\right\| \leq\left\|\tilde{\omega}_{\Theta}\right\|\left[\frac{1}{2 \gamma_{8}}+\frac{\gamma_{8}}{2}\left\|z_{2_{\Theta}}\right\|^{2}\right]$. Therefore, there exist sufficiently small positive constants $\lambda, \gamma_{i}$, $i=1, \ldots, 9$, and sufficiently large positive constants $\kappa_{j}$, $j=1, \ldots, 5$ such that

$$
\begin{aligned}
\dot{V}_{3} \leq & -\lambda V_{3}+\left[\kappa_{1} \beta_{v_{\Theta}}(\cdot)\left\|\tilde{v}_{\Theta}\right\|+\kappa_{2} \beta_{\omega_{\Theta}}(\cdot)\left\|\tilde{\omega}_{\Theta}\right\|\right] V_{3} \\
& +\kappa_{3}\left\|\mathbf{e}_{\Theta}\right\|^{2}+\kappa_{4} \beta_{v_{\Theta}}(\cdot)\left\|\tilde{v}_{\Theta}\right\|+\kappa_{5} \beta_{\omega_{\Theta}}(\cdot)\left\|\tilde{\omega}_{\Theta}\right\| \\
& +\frac{1}{2 \gamma_{1}}\left\|\delta_{\Theta}\right\|^{2}
\end{aligned}
$$

where in view of (29)-(30), the signals $\kappa_{1} \beta_{1_{\Theta}}(\cdot)\left\|\tilde{v}_{\Theta}\right\|$, $\kappa_{2} \beta_{\omega_{\Theta}}(\cdot)\left\|\tilde{\omega}_{\Theta}\right\|, \kappa_{4} \beta_{v_{\Theta}}(\cdot)\left\|\tilde{v}_{\Theta}\right\|, \kappa_{5} \beta_{\omega_{\Theta}}(\cdot)\left\|\tilde{\omega}_{\Theta}\right\|$ are $\mathcal{L}_{1}$ defined on $\left[T^{\dagger}, T\right)$, and $\kappa_{3}\left\|\mathbf{e}_{\Theta}\right\|^{2}$ is $\mathcal{L}_{2}$ defined on $\left[T^{\dagger}, T\right)$. Applying the Comparison Lemma to (44) and using [46, Lemma 1] we conclude that $V_{3}$ is bounded on $\left[T^{\dagger}, T\right)$. Moreover, when $T=+\infty, V_{3}$ converges to a ball of radius $\frac{\left\|\delta_{\Theta}\right\|^{2}}{2 \lambda \gamma_{1}}$ as $t \rightarrow \infty$. Standard signal chasing arguments can now be applied to conclude that the signals $\hat{v}_{\Theta}(t), \hat{\omega}_{\Theta}(t), u_{\Theta}(t)$ and $e(t)$ in closed-loop system remain bounded. Furthermore, applying the same arguments described in the proof of item ii) of Theorem 1, one conclude that the tracking error $\left\|p(t)-p_{d}(t)\right\|$ converges to a neighborhood of the origin that can be made arbitrarily small.

\section{Derivation of (35)}

Using (16) for $\Theta=\Theta^{\star}$ and the fact that $v=\hat{v}_{\Theta^{*}}-\tilde{v}_{\Theta^{*}}=$ $\hat{v}_{\Theta^{\dagger}}-\tilde{v}_{\Theta^{\dagger}}$, and $\omega=\hat{\omega}_{\Theta^{*}}-\tilde{\omega}_{\Theta^{*}}=\hat{\omega}_{\Theta^{\dagger}}-\tilde{\omega}_{\Theta^{\dagger}}$, the timederivative of $V$ in (34) can be written as

$$
\begin{aligned}
& \dot{V}=-\hat{v}_{\Theta^{*}}^{\prime} \mathbf{M} L_{v} \hat{v}_{\Theta^{\star}}-\hat{\omega}_{\Theta^{\star}}^{\prime} \mathbf{J} L_{\omega} \hat{\omega}_{\Theta^{*}} \\
& +\hat{v}_{\Theta^{\star}}^{\prime}\left[-S\left(\hat{\omega}_{\Theta^{\dagger}}-\tilde{\omega}_{\Theta^{\dagger}}\right) \mathbf{M} \hat{v}_{\Theta^{\dagger}}+S(\omega) \mathbf{M} \tilde{v}_{\Theta^{\dagger}}\right. \\
& +f_{v}\left(\hat{v}_{\Theta^{\dagger}}, p, R\right)+f_{v}(v, p, R)-f_{v}\left(\hat{v}_{\Theta^{\dagger}}, p, R\right) \\
& +g_{v}\left[\begin{array}{ll}
1 & 0_{1 \times 3}
\end{array}\right] \mathcal{K}_{\Theta^{\dagger}}(\cdot)+\mathbf{M} L_{v}\left(\hat{v}_{\Theta^{\dagger}}-\tilde{v}_{\Theta^{\dagger}}\right) \\
& \left.-\beta_{v_{\Theta^{*}}}\left(\hat{v}_{\Theta^{*}}, \hat{\omega}_{\Theta^{*}}, v, \omega, p, R\right) \mathbf{M} \tilde{v}_{\Theta^{\star}}\right] \\
& +\hat{\omega}_{\Theta^{\star}}^{\prime}\left[-S\left(\hat{v}_{\Theta^{\dagger}}-\tilde{v}_{\Theta^{\dagger}}\right) \mathbf{M}\left(\hat{v}_{\Theta^{\dagger}}-\tilde{v}_{\Theta^{\dagger}}\right)-S\left(\hat{\omega}_{\Theta^{\dagger}}\right.\right. \\
& \left.-\tilde{\omega}_{\Theta^{\dagger}}\right) \mathbf{J}\left(\hat{\omega}_{\Theta^{\dagger}}-\tilde{\omega}_{\Theta^{\dagger}}\right)+f_{\omega}\left(\hat{v}_{\Theta^{\dagger}}, \hat{\omega}_{\Theta^{\dagger}}, p, R\right) \\
& +f_{\omega}(v, \omega, p, R)-f_{\omega}\left(\hat{v}_{\Theta^{\dagger}}, \omega, p, R\right)+f_{\omega}\left(\hat{v}_{\Theta^{\dagger}}, \omega, p, R\right) \\
& -f_{\omega}\left(\hat{v}_{\Theta^{\dagger}}, \hat{\omega}_{\Theta^{\dagger}}, p, R\right)+G_{\omega}\left[0_{3 \times 1} I_{3 \times 3}\right] \mathcal{K}_{\Theta^{\dagger}}(\cdot) \\
& \left.-\mathbf{J} L_{\omega}\left(\hat{\omega}_{\Theta^{\star}}-\tilde{\omega}_{\Theta^{\star}}\right)-\beta_{\omega_{\Theta^{\star}}}\left(\hat{v}_{\Theta^{\star}}, \hat{\omega}_{\Theta^{\star}}, \omega, p, R\right) \mathbf{J} \tilde{\omega}_{\Theta^{\star}}\right] \text {. }
\end{aligned}
$$

Using the Young's inequality, after a straightforward but messy computations, one can conclude that there exist a sufficiently small $\lambda>0$ and sufficiently large $\kappa_{j}>0, j=1, \ldots, 13$ such that for all $t \in\left[T^{\dagger}, T\right)$, inequality (35) holds with

$$
\begin{aligned}
a_{1}(t)= & \kappa_{2} \beta_{1_{\Theta^{\dagger}}}\left(\hat{v}_{\Theta^{\dagger}}, v, \omega, p, R\right)\left\|\tilde{v}_{\Theta^{\dagger}}\right\|+\kappa_{3} \beta_{v_{\Theta^{*}}}\left(\hat{v}_{\Theta^{*}}, \hat{\omega}_{\Theta^{*}},\right. \\
& v, \omega, p, R)\left\|\tilde{v}_{\Theta^{*}}\right\|+\kappa_{4} \beta_{2_{\Theta^{\dagger}}}\left(\hat{v}_{\Theta^{\dagger}}, \hat{\omega}_{\Theta^{\dagger}}, v, \omega, p, R\right)\left\|\tilde{v}_{\Theta^{\dagger}}\right\|
\end{aligned}
$$




$$
\begin{aligned}
& +\kappa_{5} \beta_{\omega_{\Theta^{\dagger}}}\left(\hat{v}_{\Theta^{\dagger}}, \hat{\omega}_{\Theta^{\dagger}}, \omega, p, R\right)\left\|\tilde{\omega}_{\Theta^{\dagger}}\right\| \\
& +\kappa_{6} \beta_{\omega_{\Theta^{\star}}}\left(\hat{v}_{\Theta^{\star}}, \hat{\omega}_{\Theta^{\star}}, v, \omega, p, R\right)\left\|\tilde{\omega}_{\Theta^{\star}}\right\| \\
a_{2}(t)= & \kappa_{7}\left\|\tilde{\mathbf{e}}_{\Theta^{\dagger}}\right\|^{2} \\
b_{1}(t)= & \kappa_{8} \beta_{1_{\Theta^{\dagger}}}\left(\hat{v}_{\Theta^{\dagger}}, v, \omega, p, R\right)\left\|\tilde{v}_{\Theta^{\dagger}}\right\|+\kappa_{9} \beta_{v_{\Theta^{\star}}}\left(\hat{v}_{\Theta^{\star}}, \hat{\omega}_{\Theta^{\star}},\right. \\
& v, \omega, p, R)\left\|\tilde{v}_{\Theta^{\star}}\right\|+\kappa_{10} \beta_{\Theta_{\Theta^{\dagger}}}\left(\hat{v}_{\Theta^{\dagger}}, \hat{\omega}_{\Theta^{\dagger}}, v, \omega, p, R\right)\left\|\tilde{v}_{\Theta^{\dagger}}\right\| \\
& +\kappa_{11} \beta_{\omega_{\Theta^{\dagger}}}\left(\hat{v}_{\Theta^{\dagger}}, \hat{\omega}_{\Theta^{\dagger}}, \omega, p, R\right)\left\|\tilde{\omega}_{\Theta^{\dagger}}\right\| \\
& +\kappa_{12} \beta_{\omega_{\Theta^{\star}}}\left(\hat{v}_{\Theta^{\star}}, \hat{\omega}_{\Theta^{\star}}, v, \omega, p, R\right)\left\|\tilde{\omega}_{\Theta^{\star}}\right\|, \\
b_{2}(t)= & \kappa_{13}\left\|\tilde{\mathbf{e}}_{\Theta^{\dagger}}\right\|^{2} .
\end{aligned}
$$

\section{REFERENCES}

[1] H. Goldstein, Classical Mechanics, $2^{\text {nd }}$ ed. Reading, MA, USA: Addison-Wesley, 1980, 672 pages.

[2] J. T.-Y. Wen, "Control of nonholonomic systems," in The Control Handbook, W. S. Levine, Ed. Florida, USA: CRC Press \& IEEE Press, 1996, pp. 1359-1368.

[3] M. Reyhanoglu, A. van der Schaft, N. H. McClamroch, and I. Kolmanovsky, "Dynamics and control of a class of underactuated mechanical systems," IEEE Trans. on Automat. Contr., vol. 44, no. 9, pp. 16631671, 1999.

[4] F. Bullo, N. E. Leonard, and A. D. Lewis, "Controllability and motion algorithms for underactuated Lagrangian systems on Lie groups," IEEE Trans. on Automat. Contr., vol. 45, no. 8, pp. 1437-1454, 2000.

[5] J. S. Shamma and J. R. Cloutier, "Gain-scheduled missile autopilot design using linear parameter varying transformations," J. of Guidance, Control, and Dynamics, vol. 16, no. 2, pp. 256-263, 1993.

[6] I. Kaminer, A. Pascoal, E. Hallberg, and C. Silvestre, "Trajectory tracking controllers for autonomous vehicles: An integrated approach to guidance and control," J. of Guidance, Control, and Dynamics, vol. 21, no. 1, pp. 29-38, 1998.

[7] W. J. Rugh and J. S. Shamma, "Research on gain-scheduling," Automatica, vol. 36, no. 10, pp. 1401-1425, 2000.

[8] T. Koo and S. Sastry, "Output tracking control design of a helicopter model based on approximate linearization," in Proc. of the 37th Conf. on Decision and Contr., Tampa, FL, USA, Dec. 1998, pp. 3635-3640.

[9] S. Al-Hiddabi and N. McClamroch, "Tracking and maneuver regulation control for nonlinear nonminimum phase systems: application to flight control," IEEE Trans. on Contr. Systems Tech., vol. 10, no. 6, pp. 780792, 2002.

[10] J. R. T. Lawton, R. W. Beard, and B. J. Young, "A decentralized approach to formation maneuvers," IEEE Trans. Robot. Automat., vol. 19, no. 6, pp. 933-941, 2003.

[11] A. Isidori, Nonlinear Control Systems, $3^{\text {rd }}$ ed. London, UK: SpringerVerlag, 1989.

[12] J. M. Godhavn, "Nonlinear tracking of underactuated surface vessels," in Proc. of the 35th Conf. on Decision and Contr., Kobe, Japan, Dec. 1996, pp. 975-980.

[13] K. Y. Pettersen and H. Nijmeijer, "Global practical stabilization and tracking for an underactuated ship - a combined averaging and backstepping approach," in Proc. IFAC Conf. on Systems Structure and Control, Nantes, France, July 1998, pp. 59-64.

[14] F. Alonge, F. D'Ippolito, and F. Raimondi, "Trajectory tracking of underactuated underwater vehicles," in Proc. of the 40th Conf. on Decision and Contr., Orlando, Florida, USA, Dec. 2001.

[15] Z.-P. Jiang, "Global tracking control of underactuated ships by Lyapunov's direct method," Automatica, vol. 38, pp. 301-309, 2002.

[16] A. Behal, D. Dawson, W. Dixon, and Y. Fang, "Tracking and regulation control of an underactuated surface vessel with nonintegrable dynamics," IEEE Trans. on Automat. Contr., vol. 47, no. 3, pp. 495-500, Mar. 2002.

[17] K. D. Do, Z. P. Jiang, and J. Pan, "Underactuated ship global tracking under relaxed conditions," IEEE Trans. on Automat. Contr., vol. 47, no. 9, pp. 1529-1536, Sept. 2002.

[18] — - "Universal controllers for stabilization and tracking of underactuated ships," Syst. \& Contr. Lett., vol. 47, pp. 299-317, 2002.

[19] K. Y. Pettersen and H. Nijmeijer, "Tracking control of an underactuated ship," IEEE Trans. on Contr. Systems Tech., vol. 11, no. 1, pp. 52-61, 2003.

[20] E. Frazzoli, M. Dahleh, and E. Feron, "Trajectory tracking control design for autonomous helicopters using a backstepping algorithm," in Proc. of the 2000 Amer. Contr. Conf., Chicago, IL, USA, June 2000.
[21] J. P. Hespanha, D. Liberzon, and A. S. Morse, "Supervision of integralinput-to-state stabilizing controllers," Automatica, vol. 38, no. 8, pp. $1327-1335,2002$.

[22] M. Krstić, I. Kanellakopoulos, and P. Kokotović, Nonlinear and Adaptive Control Design. New York, USA: John Wiley \& Sons, Inc., 1995.

[23] S. Sastry and M. Bodson, Adaptive Control: Stability, Convergence, and Robustness. Englewood Cliffs, NJ: Prentice-Hall, 1989.

[24] P. A. Ioannou and J. Sun, Robust Adaptive Control. Englewood Cliffs, NJ: Prentice-Hall, 1996.

[25] J. P. Hespanha, D. Liberzon, and A. S. Morse, "Overcoming the limitations of adaptive control by means of logic-based switching," Syst. \& Contr. Lett., vol. 49, no. 1, pp. 49-65, 2003.

[26] A. S. Morse, "Supervisory control of families of linear set-point controllers, part 1: Exact matching," IEEE Trans. on Automat. Contr., vol. 41, no. 10, pp. 1413-1431, 1996.

[27] J. P. Hespanha and A. S. Morse, "Certainty equivalence implies detectability," Syst. \& Contr. Lett., vol. 36, no. 1, pp. 1-13, 1999.

[28] J. P. Hespanha, D. Liberzon, and A. S. Morse, "Logic-based switching control of a nonholonomic system with parametric modeling uncertainty," Syst. \& Contr. Lett., vol. 38, no. 3, pp. 167-177, 1999.

[29] G. Chang, J. Hespanha, A. S. Morse, M. Netto, and R. Ortega, "Supervisory field-oriented control of induction motors with uncertain rotor resistance," Int. J. of Adapt. Control and Signal Process., vol. 15, no. 3, pp. 353-375, 2001.

[30] D. Angeli and E. Mosca, "Lyapunov-based switching supervisory control of nonlinear uncertain systems," IEEE Trans. on Automat. Contr., vol. 47, no. 3, pp. 500-505, 2002.

[31] F. Bullo, "Stabilization of relative equilibria for underactuated systems on riemannian manifolds," Automatica, vol. 36, pp. 1819-1834, 2000.

[32] C. Samson, "Path-following and time-varying feedback stabilization of a wheeled mobile robot," in Proc. of the ICARCV 92, Singapore, 1992, pp. RO-13.1.1-RO-13.1.5.

[33] C. C. d. Wit, H. Khennouf, C. Samson, and O. J. Sordalen, "Nonlinear control design for mobile robots," in Recent Trends in Mobile Robots, Y. F. Zheng, Ed., vol. 11. World Scientific Series in Robotics and Automated Systems, 1993, pp. 121-156.

[34] C. Samson, "Control of chained systems: Application to path following and time-varying point-stabilization of mobile robots," IEEE Trans. on Automat. Contr., vol. 40, no. 1, pp. 64-77, 1995.

[35] Z.-P. Jiang and H. Nijmeijer, "A recursive technique for tracking control of nonholonomic systems in chained form," IEEE Trans. on Automat. Contr., vol. 44, no. 2, pp. 265-279, 1999.

[36] C. Altafini, "Following a path of varying curvature as an output regulation problem," IEEE Trans. on Automat. Contr., vol. 47, no. 9, pp. 1551-1556, 2002.

[37] J. Hauser and R. Hindman, "Aggressive flight maneuvers," in Proc. of the 36th Conf. on Decision and Contr., San Diego, CA, USA, Dec. 1997, pp. 4186-4191.

[38] P. Encarnação and A. M. Pascoal, "3D path following control of autonomous underwater vehicles," in Proc. of the 39th Conf. on Decision and Contr., Sydney, Australia, Dec. 2000.

[39] R. Skjetne, T. I. Fossen, and P. Kokotović, "Robust output maneuvering for a class of nonlinear systems," Automatica, vol. 40, no. 3, pp. 373383, 2004.

[40] A. P. Aguiar, D. B. Dačić, J. P. Hespanha, and P. Kokotović, "Pathfollowing or reference-tracking? An answer based on limits of performance," in Proc. of the 5th IFAC/EURON Symp. on Intel. Auton. Vehic.,, Lisbon, Portugal, July 2004.

[41] A. P. Aguiar, J. P. Hespanha, and P. Kokotović, "Path-following for non-minimum phase systems removes performance limitations," IEEE Trans. on Automat. Contr., vol. 50, no. 2, pp. 234-239, 2005.

[42] A. P. Aguiar and J. P. Hespanha, "Position tracking of underactuated vehicles," in Proc. of the 2003 Amer. Contr. Conf., Denver, CO, USA, June 2003.

[43] A. P. Aguiar, L. Cremean, and J. P. Hespanha, "Position tracking for a nonlinear underactuated hovercraft: Controller design and experimental results," in Proc. of the 42nd Conf. on Decision and Contr., Hawaii, USA, Dec. 2003.

[44] A. P. Aguiar and J. P. Hespanha, "Logic-based switching control for trajectory-tracking and path-following of underactuated autonomous vehicles with parametric modeling uncertainty," in Proc. of the 2004 Amer. Contr. Conf., Boston, MA, USA, June 2004.

[45] H. K. Khalil, Nonlinear Systems, $2^{\text {nd }}$ ed. New Jersey, USA: PrenticeHall, 1996.

[46] A. S. Morse, "Towards a unified theory of parameter adaptive control: Tunability," IEEE Trans. on Automat. Contr., vol. 35, no. 9, pp. 1002 1012, 1990. 
[47] L. Cremean, W. Dumbar, D. van Gogh, J. Hickey, E. Klavins, J. Meltzer, and R. Murray, "The caltech multi-vehicle wireless testbed," in Proc. of the 41st Conf. on Decision and Contr., Las Vegas, NV, USA, Dec. 2002.

[48] A. P. Aguiar and A. M. Pascoal, "Modeling and control of an autonomous underwater shuttle for the transport of benthic laboratories," in Proc. of the Oceans'97 Conf., Halifax, Nova Scotia, Canada, Oct. 1997.

[49] A. P. Aguiar, "Nonlinear motion control of nonholonomic and underactuated systems," Ph.D. dissertation, Dept. Electrical Engineering, Instituto Superior Técnico, IST, Lisbon, Portugal, 2002.

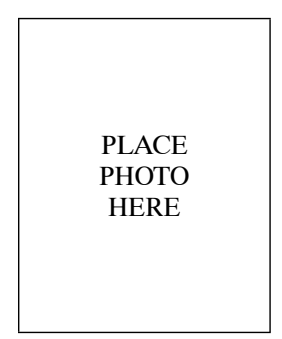

A. Pedro Aguiar received the Licenciatura, M.S. and Ph.D. in electrical and computer engineering from the Instituto Superior Tcnico, Technical University of Lisbon, Portugal in 1994, 1998 and 2002, respectively. From 2002 to 2005, he was a post-doctoral researcher at the Center for Control, Dynamical-Systems, and Computation at the University of California, Santa Barbara. Currently, Dr. Aguiar holds an Invited Assistant Professor position with the Department of Electrical and Computer Engineering, Instituto Superior Tcnico, and a Senior Researcher position with the Institute for Systems and Robotics, Instituto Superior Tcnico (ISR/IST).

His research interests include modeling, control, navigation, and guidance of autonomous vehicles; nonlinear control; switched and hybrid systems; tracking, path-following; performance limitations; nonlinear observers; the integration of machine vision with feedback control; and coordinated/cooperative control of multiple autonomous robotic vehicles.

Further information related to Dr. Aguiar's research can be found at http://users.isr.ist.utl.pt/ pedro.

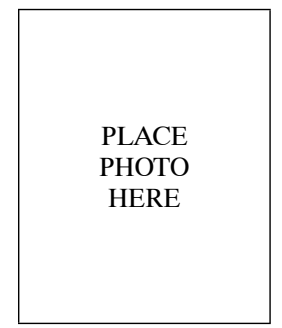

João P. Hespanha received the Licenciatura in electrical and computer engineering from the Instituto Superior Técnico, Lisbon, Portugal in 1991 and the M.S. and Ph.D. degrees in electrical engineering and applied science from Yale University, New Haven, Connecticut in 1994 and 1998, respectively. He currently holds an Associate Professor position with the Department of Electrical and Computer Engineering, the University of California, Santa Barbara. From 1999 to 2001, he was an Assistant Professor at the University of Southern California, Los Angeles. Dr. Hespanha is the associate director for the Center for Control, Dynamicalsystems, and Computation (CCDC) and an executive committee member for the Institute for Collaborative Biotechnologies (ICB), an Army sponsored University Affiliated Research Center (UARC).

His research interests include hybrid and switched systems; the modeling and control of communication networks; distributed control over communication networks (also known as networked control systems); the use of vision in feedback control; stochastic modeling in biology; and the control of haptic devices. He is the author of over one hundred technical papers and the PI and co-PI in several federally funded projects.

Dr. Hespanha is the recipient of the Yale Universitys Henry Prentiss Becton Graduate Prize for exceptional achievement in research in Engineering and Applied Science, a National Science Foundation CAREER Award, the 2005 Automatica Theory/Methodology best paper prize, and the best paper award at the 2nd Int. Conf. on Intelligent Sensing and Information Processing. Since 2003, he has been an Associate Editor of the IEEE Transactions on Automatic Control.

More information about Dr. Hespanha's research can be found at http://www.ece.ucsb.edu/ hespanha. 